\title{
Standardization of electrolyte leakage data and a novel liquid nitrogen control improve measurements of cold hardiness in woody tissue
}

Alisson Pacheco Kovaleski ( $\square$ al.kovaleski@wisc.edu )

University of Wisconsin-Madison https://orcid.org/0000-0002-3287-4963

Jake J Grossman

Swarthmore College

\section{Methodology}

Keywords: Acer, cold hardiness, differential thermal analysis, electrolyte leakage, freezing tolerance, maple

Posted Date: April 7th, 2021

DOl: https://doi.org/10.21203/rs.3.rs-392368/v1

License: (c) (i) This work is licensed under a Creative Commons Attribution 4.0 International License.

Read Full License 
Article Title

2 Standardization of electrolyte leakage data and a novel liquid nitrogen control improve measurements of

3 cold hardiness in woody tissue

5 Authors (*authors contributed equally)

$6 \quad *$ Alisson P. Kovaleski

7 Arnold Arboretum of Harvard University, 1300 Centre St., Boston, MA 02131, USA

8 Department of Horticulture, University of Wisconsin-Madison, 1575 Linden Drive, Madison, WI 53706, 9 USA

10 Corresponding author: al.kovaleski@wisc.edu

*Jake J. Grossman

13 Arnold Arboretum of Harvard University, 1300 Centre St., Boston, MA 02131, USA

14 Biology Department, Swarthmore College, 500 College Ave., Swarthmore, PA 19081, USA

\section{Author Contributions}

17 A.P.K. and J.J.G. jointly and equally conceived of the original project idea, designed the experiment, performed all field and laboratory work, and analyzed resultant data. Both authors contributed to all drafts of the manuscript. Both authors agree to be responsible for contact and ensure communication.

Funding Information Arboretum of Harvard University.

\section{Abstract}

Background. A variety of basic and applied research programs in plant biology require the accurate and

27 reliable determination of plant tissue cold hardiness. Over the past 50 years, the electrolyte leakage

28 method has emerged as a popular and practical method for quantifying the amount of damage inflicted on

29 plant tissue by exposure to freezing temperatures. Numerous approaches for carrying out this method and

30 analyzing the resultant data have emerged. These include multiple systems for standardizing and

31 modeling raw electrolyte leakage data and multiple protocols for boiling samples in order to maximize

32 leakage as a positive control. We compare four different routines for standardization of leakage data and

33 assess a novel control method - immersion in liquid nitrogen in lieu of traditional boiling - and apply

34 them to woody twigs collected from 12 maple (Acer) species in early spring. We compare leakage data

35 from these samples using each of four previously published forms of data analysis and boiling vs. liquid 
nitrogen controls and validate each of these approaches against visual estimates of freezing damage and

37 differential thermal analysis.

38 Results. Through presentation of our own data and re-analysis of previously published findings, we show

39 that standardization of raw data against estimates of both minimum and maximum attainable freezing

40 damage allows for reliable estimation of cold hardiness at the species level and across studies in diverse

41 systems. Furthermore, use of our novel liquid nitrogen control produces data commensurate across studies

42 and enhances the consistency and realism of the electrolyte leakage method, especially for very cold

43 hardy samples.

44 Conclusion. Future leakage studies that relativize data against minimum and maximum leakage and that

45 employ our updated liquid nitrogen control will contribute generalizable, repeatable, and realistic data to

46 the existing body of cold hardiness research in woody plants. Data from studies conducted using a liquid

47 nitrogen (and not a boiling) control can still be compared to previously published data, especially when

48 raw data are standardized using the best-performing approach among those we assessed. Electrolyte

49 leakage of woody twigs emerges as a useful technique for quickly assessing the probability of tissue death

50 in response to freezing in dormant plants. Differential thermal analysis may provide different and

51 complementary information on cold hardiness.

\section{Keywords}

54 Acer, cold hardiness, differential thermal analysis, electrolyte leakage, freezing tolerance, maple

\section{$56 \quad$ Background}

57 How do plants differ in their cold hardiness, the ability to avoid or tolerate exposure to temperatures

58 below freezing [1]? The capacity to withstand freezing temperatures structures global plant distribution,

59 determining which lineages can radiate into high-latitude or -altitude environments [2,3]. Within these

60 environments, plants that can withstand not just freezing, but extreme cold, are able to realize a larger

61 niche than those more vulnerable to frost or freezing [4,5]. In short, cold hardiness shapes the ecological 
62 dynamics and evolutionary trajectory of temperate plant evolution. Across research domains, researchers

63 require tools for evaluating cold hardiness that can be efficiently repeated across diverse plant lineages

64 while providing interpretable, mechanistic evaluations of cold hardiness traits.

66 In the years since 1932 when Dexter and colleagues' [6] observation that freezing temperatures

67 destabilize the cellular membrane and accelerate leakage of symplastic solutes out of the cell,

68 quantification of electrolyte leakage has emerged as a tool for quantifying cold hardiness. Flint et al. [7]

69 subsequently developed the Index of Injury $(I)$ approach, which is calculated through comparison of

70 samples incubated in pure water after being frozen. Values of $I$ close to 0 indicate very little disruption of

71 cellular stability and consequently little leakage while higher values indicate greater disruption and more

72 leakage. Over the past 50 years, the measurement of electrolyte leakage from stem and leaf tissues of

73 woody plants adapted to cold climates has remained popular (reviewed in the Introduction of [8]; see [9-

74 11] for more recent examples). Despite this, there exists considerable diversity in the benchtop and

75 analytical approaches employed in electrolyte leakage measurements. We carried out the present work

76 with a particular focus on variability in two areas: the analytical procedures for extraction of critical cold

77 hardiness values from electrolyte leakage data and the control treatment used to relativize freezing

78 damage.

80 How is cold hardiness determined from leakage?

81 Since the development of the electrolyte leakage method, practitioners have employed diverse approaches

82 to determine cold hardiness from raw electrolyte leakage data. Typically, procedures follow a three-step

83 approach: absolute electrolyte leakage of a sample is made relative $(R)$ by normalizing against electrolyte

84 leakage at an extreme control (typically boiling, as discussed further below), followed by optional

85 adjustment that results in $I$, and curve fitting, which allows extraction of a critical cold hardiness value.

86 Variation in the choices made at each of these steps has produced variation in the ways that leakage data

87 are interpreted in the literature (Fig. 1). 
89 At the first stage of processing electrolyte leakage data, once $R$ is calculated for a given individual across

90 a range of freezing temperatures, it can be transformed into $I$ by "zeroing" against unfrozen control

91 samples and $I_{a d j}$ by also "stretching" relative to a theoretical maximum level of damage. In their work,

92 Kreyling et al. [10] opt to do neither; they simply fit curves of $R$ (REL in their notation) vs. freezing

93 temperature across a wide range of temperatures $\left(5^{\circ} \mathrm{C}\right.$ to $\left.-196^{\circ} \mathrm{C}\right)$. In this case, $R$ can range from 0 to

$94100 \%$, but these values are rarely attained. Flint and colleagues' [7] approach is similar, except that $R$

95 values are zeroed against a negative control kept at room temperature to take into account any electrolyte

96 leakage from samples that might occur due to handling and processing unrelated to freezing (e.g., cut

97 ends of stem pieces), thus becoming I. Curves fit to these data therefore treat freezing damage

98 commensurate with handling and processing damage as " $0 \%$ " damage by default. Finally, Lim and

99 colleagues [12], popularized an approach that goes one step further, stretching all values of $I$ prior to

100 curve fitting such that a sample's leakage at the lowest temperature tested (often at or below $-80^{\circ} \mathrm{C}$ ) is

101 treated as the maximum possible damage for that sample, giving $I_{a d j}$. Curves fit using this procedure

102 contain values of $I$ corresponding to $0 \%$ and $100 \%$ damage. These three approaches represent a gradient

103 in adjustment of raw electrolyte leakage values ranging from none ("Kreyling”) to zeroing against a

104 minimum ("Flint") to both zeroing and stretching to a maximum ("Lim").

105

106 Second, curves are fit to one of several curves chosen to model progressive accumulation of damage with 107 exposure to freezing. Historically, most authors have fit data to generalized logistic curves [13-17], but

108 some may use linear models as well [11]. However, while such logistic models always represent damage 109 in response to dropping temperatures as a symmetric, sigmoidal process, actual plant tissues may accrue 110 damage asymmetrically. This is to say, for instance, that marginal increases in damage may occur much 111 more rapidly (over smaller temperature intervals) prior to the inflection point of the sigmoid curve than 112 after it. For this reason, Von Fircks and Verwijst [18] first suggested using the Richards function, which 113 can produce asymmetric, sigmoidal curves, to model $I$ over a range of temperatures. Subsequent work 
114 [8a] has validated this approach, with Lim and colleagues [12] recommending the Gompertz function - a

115 special case of the Richards with one fewer parameter - as the best fit for leakage data. Many subsequent

116 workers have followed their example and modeled leakage data with the Gompertz function [9,19-21].

117 As such data processed using the Kreyling, Flint, or Lim approach can then be modeled within either a

118 logistic or Gompertz framework.

120 Third, and finally, a critical value of freezing damage, used to approximate cold hardiness, is extracted

121 from model fit to leakage data. For general logistic models, this usually corresponds to $T_{50}$, the

122 temperature at which $50 \%$ of maximum possible electrolyte leakage is attained, although other damage

123 thresholds can be adopted as well (eg., $T_{20}, T_{80}$, etc.). Yet modeling of leakage data with the asymmetric

124 Richards and Gompertz curves has also made it possible to extract a potentially more biologically realistic

125 critical value from the resulting fitted curves. Whereas the inflection point of a logistic curve, $T_{\max }$,

126 always corresponds with $T_{50}$, these two points are decoupled in asymmetric sigmoid curves. As such,

$127 T_{\max }$, the temperature at which the rate of increase in freezing-induced damage is maximized (the

128 temperature of maximum instantaneous slope of the curve), does not have to co-occur with the

129 temperature at which half of the maximum damage possible has been reached. And though $T_{\max }$ has been

130 employed in place of $T_{50}$ in the literature $[8,12]$ validation of this choice with reference to other cold

131 hardiness metrics has been limited. As such, differences in the biological realism and thus desirability of

132 the two critical values are still unclear.

133

134 What is the best way to standardize measurements of freezing-induced leakage?

135 Electrolyte leakage data have historically been standardized at the level of the individual sample to

136 control for variability among samples in size and intrinsic (e.g., species- or genotype-dependent)

137 electrolyte leakage capacity. In this work, we also assess the realism and consistency of the most

138 commonly used positive control technique. Typically, all samples are heat-killed [22] following the first 
conductivity reading, often through boiling induced by the liquid cycle of an autoclave. Yet, as a control

140 for measurements of cold hardiness, boiling fails to mimic the process of interest: freezing-induced

141 damage. In this vein, at least two groups $[8,12]$ have used conductivity following freezing at $-80^{\circ} \mathrm{C}$ in lieu

142 of boiling as an index of maximum leakage following damage to sampled tissues. However, it has

143 remained unclear whether freezing at these temperatures is actually sufficient to relativize leakage across

144 samples varying in size and anatomy [23].

145

146 Furthermore, the temperature at which samples are boiled or heated and/or the time during which this

147 temperature is reached are frequently not specified ([10,14, 24-26], among others). And though boiling at

$148120 / 121^{\circ} \mathrm{C}$ is typical (e.g., $\left.[9,17,27]\right)$, some [28-31] report using temperatures lower than the boiling

149 point of water to heat kill samples. Yet Deans et al. [13] find that even temperatures above the boiling

150 point (they compare $105^{\circ}$ vs. $121^{\circ} \mathrm{C}$ ) vary in their capacity to induce electrolyte leakage. This variability

151 may be especially problematic for woody stem tissue (as opposed to bud or dissected vascular samples),

152 from which leakage may be constrained to cut ends rather than from sample's entire surface area. In short,

153 because electrolyte leakage depends on the temperature of and duration of and time elapsing following

154 heat-killing [13,32], boiling as a control strikes us as a frequent and consequential source of inconsistency

155 in the method.

157 Our approach

158 Despite some helpful methodological comparisons [12,13,19], practitioners collecting electrolyte leakage 159 data are still confronted with numerous choices: what type of standardizing control should be employed

160 during data collection? Should data be analyzed using the Kreyling, Flint, or Lim approach to zeroing and 161 stretching? Which critical value should be extracted? And, once such decisions are made, will the 162 resulting estimates of cold hardiness actually predict field plant performance? 
164 In the work presented here, we respond to existing diversity in the methods used to assess cold hardiness

165 through electrolyte leakage by: (i) integrating novel measurements in a panel of 12 maple (Acer spp.)

166 species (Fig. 2), and (ii) re-analyzing published data. Our aim is to consolidate existing methods in

167 measuring leakage, validate these methods against other techniques for measuring cold hardiness, and

168 suggest a standard operating procedure in contrast to the variable status quo. We focus on two main

169 domains in which existing practice could be improved. First, we contrast the performance of four main

170 approaches to processing maple electrolyte leakage data: logistic modeling of data adjusted following 1)

171 Kreyling et al. [10], 2) Flint et al. [7], and 3) Lim et al. [12] and 4) Gompertz modeling of data adjusted

172 following the Lim approach. And second, we introduce a novel control procedure of liquid nitrogen

173 immersion and compare its performance to that of the standard boiling control. Our approach addresses

174 four questions:

175 1) Which of the four approaches to modeling electrolyte leakage data produce estimates of cold

176 hardiness most aligned with those from two other core approaches: visual damage and differential

177 thermal analysis?

178 2) Does use of a liquid nitrogen control

179 a. produce leakage data commensurate with those generated with a boiling control?

180 b. improve generalizability of findings across approaches to modeling leakage data?

1813 3) Does re-analysis of previously collected data $[10,11]$ using data zeroing and stretching and

182 assuming damage commensurate with a liquid nitrogen control provide more realistic estimates of

$183 \quad$ cold hardiness?

184 In presenting this analysis, we encourage other investigators to continue using electrolyte leakage to

185 measure cold hardiness and facilitate synthesis of existing and forthcoming data despite the use of diverse

186 protocols.

187

188 Results 
191 By design, the four analytical approaches we compared generated variability in curves representing the 192 relationship between electrolyte leakage ( $R$ or $I$ ) and freezing temperature (Fig. 3; model parameters 193 given in Additional file 1). Yet critical values extracted using each approach occupy a similar range of

194 values ( -12 to $-28{ }^{\circ} \mathrm{C}$; Table $\left.1 \mathrm{~A}\right)$ and were generally correlated with each other (Table 2$)$; species that had 195 already broken flower or leaf buds were generally less cold hardy than those that still appeared dormant 196 (Table 1A). Across methods, leakage measurements generated a warmer and somewhat narrower range of 197 critical values relative to visual damage estimates $\left(-17\right.$ to $\left.-36{ }^{\circ} \mathrm{C}\right)$ and LTEs $\left(-20\right.$ to $-38{ }^{\circ} \mathrm{C}$, Table $\left.1 \mathrm{~A}\right)$.

199 Yet all four approaches to modeling electrolyte leakage did not perform equally well in predicting critical 200 temperatures for freezing damage as measured using these other methodologies (Fig. 4, Table 2). Across a 201 range of critical visual damage levels $(20 \%, 50 \%$, and $80 \%), T_{50}$ extracted from general logistic curves fit 202 using the Lim approach best predicted visual damage $\left(T_{50}\right.$ or $\left.T_{80}\right)$ and species differences in LTEs.

203 Critical values extracted using the Lim approach with Gompertz (rather than general logistic) curve fitting 204 also performed well, but these require secondary calculation from model parameters, and therefore are 205 less intuitive and less frequently used than general logistic curves. For these reasons, in the following 206 analyses, we present electrolyte leakage data analyzed using the Lim logistic protocol: zeroed and stretched 207 data fit to a logistic curve. Critical values for leakage, when determined using this approach, approximate 208 critical values for visually estimated damage despite diversity across species and genotypes (Fig. 5,6).

210 We found support for the convention of comparing critical temperatures at the point at which $50 \%$ of total 211 leakage or visual damage occurs (Fig. 7,8). Critical electrolyte leakage best predicts the temperatures at 212 which between 50 and $80 \%$ of visually diagnosed damage occurs, although bias and error between the 213 two assays is lower from 20 to $50 \%$ visual damage. Visual damage is accrued at a faster rate than 214 electrolyte leakage (Fig. 5; see values of the $b$ parameter in Additional file 1). This explains the 
215 minimization of bias and RMSE for their comparison in the 30-60\% range of visual damage. Therefore, a 216 comparison of $T_{50}$ for both electrolyte leakage and visual damage strikes us as an acceptable validation 217 procedure. As shown in the middle panel of Fig. 7, this comparison indicates that electrolyte leakage 218 explains $36 \%$ of the variability in visual damage and underestimates $T_{50}$ for visual damage by only $3{ }^{\circ} \mathrm{C}$.

220 Species-level LTEs show a different, though consistent, pattern of cold hardiness compared to critical 221 temperatures for either electrolyte leakage or visual damage. Ease of exotherm identification in DTA 222 differed between species, which may account for some of the differences from other methods. Ten of the 22312 study species experienced LTEs between $-34.5^{\circ}$ and $-38.4^{\circ} \mathrm{C}$, just above the theoretical supercooling 224 limit for water $\left(\sim-42^{\circ} \mathrm{C}\right.$; Table $\left.1 \mathrm{~A}\right)$. The other two species, $A$. caudatifolium and $A$. davidii, had very high 225 LTEs, indicating ice formation at roughly $-20^{\circ} \mathrm{C}$. Because of the bimodal distribution of species means in 226 LTEs, there was not a significant relationship between this critical value and $T_{50}$ for electrolyte leakage $(\rho$ $227=0.45, \mathrm{p}=0.41)$ or $T_{50}$ for visual damage $(\rho=0.31, \mathrm{p}=0.64)$.

\section{Data collected using a novel liquid nitrogen control commensurate with those collected using a boiling}

230 control

231 Electrolyte leakage induced by immersion in liquid nitrogen generated an average of $58 \%$ of the leakage 232 induced by the standard boiling approach (an autoclave cycle at $120^{\circ} \mathrm{C}$ ) and an average of $184 \%$ of the 233 leakage induced by freezing at $-80^{\circ} \mathrm{C}$. Deming regression of nitrogen-induced leakage based on boiling234 induced leakage with a zero intercept yields an allometric equation of 
238 with two outliers removed and a 95\% confidence interval around the slope ranging from 0.557 to 0.595

239 (Fig. 9). Increased variance made this relationship weaker for physically larger samples (in this case,

240 those with wider diameter), which leaked more electrolytes and had higher conductivity.

242 Use of a liquid nitrogen control provides more consistent estimates of cold hardiness across different

243 approaches to modeling leakage data

244 Allometric adjustment of leakage values $(R$ or $I)$ in which a boiling standard was used to estimate damage

245 that would have resulted from use of a liquid nitrogen standard led to a considerable improvement in the

246 consistency among approaches for converting electrolyte leakage data into estimates of cold hardiness.

247 For instance, when a boiling standard is used, leakage estimated using the Kreyling and the Lim logistic

248 approach are not commensurate above very low levels of damage (Fig. 9B, 10) - with overall bias at 51\%

249 and RMSE $=55 \%$. When leakage values are adjusted using the allometric equation presented above to

250 assume a liquid nitrogen standard, leakage estimated using the Kreyling approach increases, creating

251 greater concordance between the two approaches (Fig. 9C) - bias is on average $25 \%$ and RMSE reaches

$25229 \%$. If the two approaches are evaluated in a narrower range, between 25 and $75 \%$ of EL based on the

253 Lim $_{\text {logistic }}$ approach, bias drops to $6 \%$ for the liquid nitrogen standard vs $26 \%$ for boiling, and RMSE to

$25411 \%$ and $28 \%$ for liquid nitrogen and boiling, respectively.

256 Re-analysis of previously published data suggests overestimation of cold hardiness

257 Re-analysis of raw electrolyte leakage data from [11] underscores the consequences of variation in the 258 major approaches to analyzing data generated using this method. Each of the four approaches we used 259 returned higher critical temperatures - indicating less cold hardiness - for the oak study system than those

260 reported originally by these authors (Table 1). For instance, using the Lim logistic $_{\text {approach, we estimated }}$ 261 that unacclimated woody samples accrued $50 \%$ freezing damage between $-12^{\circ}$ and $-15^{\circ} \mathrm{C}$; originally 262 published estimates ranged from $-13^{\circ}$ and $-20^{\circ} \mathrm{C}$. Our estimated range of critical values for acclimated 
263 samples $\left(-21^{\circ}\right.$ and $\left.-25^{\circ} \mathrm{C}\right)$ was also considerably warmer than the originally reported one $\left(-29^{\circ}\right.$ and $\left.26434^{\circ} \mathrm{C}\right)$.

266 We also observed that, even though Fallon and Cavender-Bares only froze oak woody tissues to a low

267 temperature of $-40^{\circ} \mathrm{C}$, doing so appeared to cause levels of electrolyte leakage approaching or even

268 exceeding those instigated by boiling (Additional file 2). This contrasts with our findings, in which liquid

269 nitrogen immersion $\left(-200^{\circ} \mathrm{C}\right)$ only generated about $58 \%$ of the leakage caused by boiling woody tissue

270 from maples. Our figure of 58\% maximum leakage comports with those from our re-analysis of Kreyling

271 and colleagues' [10] work, in which the two surveyed maple species showed similar levels of maximum

272 leakage (58\% to $68 \%$ in March; Additional file 2).

274 Indeed, our extrapolation of findings from Kreyling and colleagues' [10] work suggests that patterns of 275 maximum freezing-induced leakage likely vary among species and over time (Additional file 2). Notably, 276 we observe that some genera, such as the redwoods (subfamily Sequoioideae) and beeches (Fagus spp.), 277 appear to release virtually all of their intracellular electrolytes when immersed in liquid nitrogen (-200 $278{ }^{\circ} \mathrm{C}$ ), while others, such as the maples and pines (Pinus spp.), resist leakage, even when unacclimated to 279 freezing. We also note a phenological pattern in maximum leakage, which, per Kreyling and colleagues' 280 findings, was highest in autumn (November), and lowest at the end of winter (February), before 281 increasing again with springtime deacclimation (March; Additional file 2). This pattern is also observed 282 in some oak species in maximum leakage in [11], where samples collected in the summer had generally 283 higher maximum leakage than in the winter - though the lowest temperature used was $-40{ }^{\circ} \mathrm{C}$. These 284 patterns suggest that the electrolyte leakage at a single temperature cannot be used to compare even the 285 same genotype across time when using boiling as a control [33] - but may be possible with a liquid 286 nitrogen control. Differences in maximum leakage among clades also suggest that further research could 287 be performed to understand what portion of electrolytes remains insoluble during freezing at liquid 288 nitrogen temperatures but becomes soluble through boiling. 
290 Neither use of short incubation times nor choice of boiling or liquid nitrogen immersion impairs estimates

291 of cold hardiness

292 Though it was not the main focus of our experiment, we assessed the sensitivity of our electrolyte leakage

293 measurements to variation in the incubation time elapsing between freezing or autoclaving and

294 measurement and to the choice of boiling (at either $100^{\circ} \mathrm{C}$ or $120^{\circ} \mathrm{C}$ ) or liquid nitrogen immersion. We

295 found that longer incubation times following experimental freezing (down to $-80^{\circ} \mathrm{C}$ ) led to higher sample

296 conductivity, but that there was no relationship between incubation time and conductivity following either

297 autoclaving or liquid nitrogen immersion (Fig. 11A, Additional file 3). In particular, conductivity

298 measured after one to two days of incubation following experimental freezing was statistically

299 indistinguishable, while conductivity continued to increase significantly on days five and seven. On the

300 other hand, post-boiling or post-liquid nitrogen conductivity did not show a significant, linear relationship

301 with incubation time.

302

303 This pattern is reflected in critical values for cold hardiness derived from these data (Additional file 4):

$304 T_{50}$ for samples from the same species and assessed using the same standardization protocol varied little 305 regardless of whether they were incubated for one or two days. Estimates of $T_{50}$ derived from samples 306 incubated for five days were generally within a few degrees of those associated with shorter incubations, 307 but, by five days, species-level differences in cold hardiness were no longer reflected in $T_{50}$ (Fig. 11B).

308 Estimates of cold hardiness from samples incubated for seven days became increasingly variable across

309 methods, potentially reflecting microbial growth on samples (Fig. 12). As such, we conclude that

310 measuring leakage for up to two days following experimental freezing does not substantially bias

311 estimates of cold hardiness, while waiting longer for leakage measurements (up to a week in our

312 comparisons) may actually produce biased or unreliable results.

313

\section{Discussion}


315 We compared late-winter cold hardiness of woody tissue in 12 maple species using our updated

316 electrolyte leakage protocol and validated these measurements against other metrics of cold hardiness. We

317 found general agreement among critical values from four distinct approaches to extracting critical cold

318 hardiness values from electrolyte leakage data, as a result of using relative parameters of fitted curves

319 rather than leakage in absolute terms (i.e., values of $R$ ). However, we recommend the use of critical

320 values associated with $50 \%$ damage obtained using the $\operatorname{Lim}_{\text {logistic }}$ approach. These critical temperatures

321 correspond well across genotype and species with the temperature at which $50 \%$ of twig tissue had

322 experienced cambial browning as determined visually. Finally, we found that liquid nitrogen immersion

323 as a control produces electrolyte leakage data commensurate with that produced with a boiling control,

324 but can be applied more consistently and is better suited to samples with low levels of maximum freezing-

325 induced leakage.

326

327

Electrolyte leakage data should be adjusted to account for minimum and maximum damage

328 In surveying the existing electrolyte leakage literature and developing this protocol, we noted

329 consequential variability in the way that raw leakage data are converted to critical values of cold

330 hardiness. As a result, it is possible to compare cold hardiness among treatment groups or species within a

331 single study, but particular values of $R$ or $I$ may not be transferable across research groups or study

332 systems. We classified the diverse published protocols into four approaches (Fig. 1) based on whether or

333 not authors standardize raw data based on minimally damaged (unfrozen) or deep-frozen (maximally

334 damaged) controls and on what class of curve is fit to adjusted data (general logistic or Gompertz).

336 Of the four resultant classes of protocols, we recommend the use of either of the Lim approaches

$337\left(\operatorname{Lim}_{\text {logistic }}\right.$ or $\left.\operatorname{Lim}_{\text {gompertz }}\right)$, in which all leakage data are converted to $I_{T, a d j}$ through zeroing against leakage

338 from non-freezing (e.g., $4^{\circ} \mathrm{C}$ ) and stretching against leakage from deep-freezing (e.g., $\left.-80^{\circ} \mathrm{C}\right)$ treatments.

339 Though we chose to fit leakage data to a general logistic curve (as in Ouyang et al., 2019a) for reasons of

340 parsimony and ease of interpretation, fitting to a Gompertz curve [12,19-21] generates similar results. 
341 Critical values obtained from this approach best predicted visually observed cambial browning across a

342 variety of damage thresholds (20-80\%; Fig. 4), including critical 50\% values (Fig. 5) across genotypes

343 and species. (However, we do note that visual browning occurred across a smaller range of temperatures

344 [Table 1]). The values of $R$ and $I$ we observed also demonstrate the need for the correction of $R$ or $I$ into

$345 I_{T, a d j}$ (i.e., using relativized measurements in the curve estimates): although in $R$ and $I$, an absolute value

346 of $50 \%$ damage was not reached, a clear plateau is observed in leakage values below $-40{ }^{\circ} \mathrm{C}$ (Fig. 3). The

347 use of absolute $50 \%$ damage $(R=50 \%)$ as the metric for cold hardiness level would have resulted in all

348 species having cold hardiness $>-80^{\circ} \mathrm{C}$ in our study, an unrealistic outcome.

350 Adoption of this approach will likely affect reporting of estimates of cold hardiness derived from

351 electrolyte leakage data. For instance, we found that critical temperatures $\left(\mathrm{LT}_{50}\right)$ for our maple samples

352 estimated using the Lim $_{\text {logistic }}$ approach were highly correlated with $\left(\mathrm{R}^{2}=0.81\right)$, but not identical to,

353 estimates generated through other approaches (Tables 1,2). And application of this approach in our re-

354 analysis of Fallon and Cavender-Bares' [11] findings produced warmer (less cold hardy) estimates of

$355 \mathrm{LT}_{50}$ by $1-9^{\circ} \mathrm{C}$ across species and degree of cold acclimation. We attribute this pattern to Fallon and

356 Cavender-Bares' [11] use of absolute $I$ values for estimation of 50\% damage (Fig. 1B). However, as

357 discussed previously, plateaus in their data indicate maximum damage may not occur at $100 \%$ of boiling

358 leakage. In fact, even at liquid nitrogen temperatures, leakage values can fall far below 100\%, and even

359 below 30\% (Additional file 2), spuriously suggesting that tissue is hardy to temperatures below $-196{ }^{\circ} \mathrm{C}$.

\section{Electrolyte leakage corresponds to visual damage, but not low temperature exotherms}

362 To further explore the biological meaningfulness of electrolyte leakage measurements, we validated

363 critical values from the Lim logistic approach against not only critical values of visual damage, but also

364 LTEs corresponding to the formation of intracellular ice. We found a generally high degree of correlation

365 between damage measured by both leakage and browning (Fig. 4), especially when leakage was between

36650 and $80 \%$ of its observed maximum (Fig. 7). For instance, across the 12 measured maple species, $\mathrm{LT}_{50}$ 
367 (calculated using the Lim ${ }_{\text {logistic }}$ approach) was highly and significantly correlated with browning $(\rho=0.58$,

$368 \mathrm{n}=36, \mathrm{p}<0.001$; Table 2). Furthermore, the ranges of these critical values also mostly overlapped, with

369 critical damage occurring between $-13^{\circ}$ and $-32^{\circ} \mathrm{C}$ (Table 1). As such, we contribute to the evidence that

370 cellular damage measured via electrolyte leakage corresponds to visually apparent damage to plant

371 cambial tissue in response to freezing [8,10,12-14,19,34,35].

373 On the other hand, we found that critical electrolyte leakage generally occurred at warmer temperatures

374 than did the formation of symplastic ice (the LTE). We documented relatively uniform LTEs ranging

375 from $-35^{\circ}$ to $-38^{\circ} \mathrm{C}$ for ten species, indicating that these trees could still prevent ice formation in their

376 cells at temperatures far below those they might ever experience in their native ranges. The other two

377 species, A. caudatifolium and A. davidii, both adapted to relatively warm East Asian climates, had high

378 LTEs $\left(-20^{\circ} \mathrm{C}\right)$. Even in these cases, LTEs occurred at temperatures below critical leakage temperatures,

379 suggesting that the two methods capture different aspects of cold hardiness. This finding is consistent

380 with past documentation that LTEs can diverge from critical values for leakage during the growing season

381 but converge when plants are fully cold-acclimated in the winter months [12,24]. As such, we find

382 evidence that visual damage, but not LTEs, should serve as a useful validation of cold hardiness methods

383 made with electrolyte leakage.

385 Liquid nitrogen as a negative control can enhance the use of the electrolyte leakage approach

386 We found that electrolyte leakage values derived from a boiling (autoclaved) control and a liquid nitrogen

387 immersion control are highly correlated $\left(\mathrm{R}^{2} \sim 80 \%\right.$; Fig. 9A). Because freezing samples at $\sim-200^{\circ} \mathrm{C}$ by

388 liquid nitrogen immersion is less damaging to woody tissue than boiling, raw leakage values calculated

389 using our new control are roughly $58 \%$ of the values calculated using a boiling control. Given all this, and

390 for the sake of transferability of findings, we conclude that values of $R$ or $I$ calculated using either method

391 can be compared across studies using the linear equation given in Fig. 9. But, since leakage data produced 
using either method are linearly related, does the choice of boiling versus liquid nitrogen immersion even matter? Based on our findings, we argue that use of liquid nitrogen bestows four advantages.

First, as surveyed above, there are a wide variety of boiling protocols used in electrolyte leakage studies; variability exists in both temperature and intended duration of boiling, likely hindering generalization of

397 findings. Furthermore, duration of boiling treatments is often not even obvious: it is unclear whether

398 duration of actual exposure to a target temperature or total time in hot water bath is reported (e.g., "killed

399 in a boiling water bath for 20 min" [36]) or an autoclave cycle (which usually also includes higher

400 pressure to prevent boiling over). Liquid nitrogen immersion, on the other hand, exposes samples to a

401 physically constrained range of temperatures $\left(-196\right.$ to $\left.-210^{\circ} \mathrm{C}\right)$ for a much more obvious duration of time

402 (the time of immersion).

404 Second, a liquid nitrogen control may be more suitable for samples collected from cold-hardy or highly 405 acclimated plants or for any sample with low levels of maximum freezing-induced leakage. Because use 406 of a boiling standard damages samples more than immersion in liquid nitrogen (Fig. 9A), the former 407 approach produces lower values of $I$ or $R$ across freezing temperatures. As a result, apparent freezing408 induced leakage measured using a boiling control often remains low, failing to reach $50 \%$ of maximum 409 damage. Low values of $R$ or $I$, in turn, are often less commensurate across different approaches to 410 calculating cold hardiness using electrolyte leakage data. For instance, in our comparison of leakage

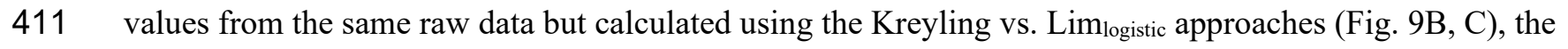
412 two approaches are only commensurate at low damage levels when data are relativized against boiling.

413 On the other hand, when data are relativized against liquid nitrogen immersion, the two approaches

414 indicate relatively similar damage across a wider range of temperatures, especially for cold-hardy samples

415 like our acclimated maples or the maples and pines in Kreyling and colleagues' [10] study (Additional file 4165 , Fig. 10). Therefore, use of a liquid nitrogen control or conversion of $R$ or $I$ to assume a liquid nitrogen 417 control facilitates comparison and synthesis across diverse approaches to measuring cold hardiness using 
418 electrolyte leakage. A caveat to this argument is our finding, through re-analysis of Fallon and Cavender-

419 Bares' [11] study of southwestern U.S. oaks, that, when maximum freezing-induced leakage is high, a

420 boiling control can be sufficient for estimation of cold hardiness using electrolyte leakage.

422 Third, we note that, as expected, liquid nitrogen immersion provides a more realistic proxy of "maximum 423 damage" to samples than did boiling. Liquid nitrogen immersion (at roughly $-200^{\circ} \mathrm{C}$ ) caused a little less

424 than twice the leakage inflicted by freezing at $-80^{\circ} \mathrm{C}$, which is already a temperature that the vast majority

425 of the world's woody plants never experience in a natural context. As such, freezing through liquid

426 nitrogen immersion seems sufficient to inflict the maximum possible damage that could be expected from

427 this mode of injury on woody samples, while avoiding the excessive treatment afforded by boiling. The

428 choice of boiling versus liquid nitrogen immersion appears to be less important for non-woody samples

429 (e.g., leaves, crowns), though may still be important for more lignified green tissues such as conifer 430 needles [37].

432 Fourth and perhaps most obviously, submerging the tubes in liquid nitrogen provides the same physical 433 environment for the samples (ice, compared to boiling liquid water) as they experience when exposed to 434 the damaging stressor of interest. Use of a liquid medium as a control means that the resulting leakage 435 occurring during boiling may be due to other effects other than only temperature.

\section{Conclusions}

438 A central recommendation we make for all workers performing electrolyte leakage research is that 439 estimates of cold hardiness using this method should take place across a range of temperatures, and not 440 simply at one freezing temperature of interest. This range must include one temperature above freezing

441 (e.g., $4^{\circ} \mathrm{C}$ ) and one or more temperatures chosen to elicit maximum freezing damage such that a plateau in 442 electrolyte leakage values is observed (e.g., $<-80^{\circ} \mathrm{C}$ and ideally including submersion in liquid nitrogen).

443 If this approach to sampling is observed, all four of the analytical approaches we surveyed are likely to 
444 yield similar critical values of cold hardiness (Tables 1,2). Generally, we found $\mathrm{LT}_{50}$, extracted from data

445 fit to either a general logistic or Gompertz curve following Lim and colleagues' [12] approach, to best

446 approximate visually observed damage to woody tissue.

448 We highly recommend the use of the liquid nitrogen control we present here in lieu of boiling or

449 autoclaving. This control will be especially useful for very cold hardy woody samples (or physically small

450 ones) coming from, for instance, acclimated temperate or boreal species prior to leaf-out. And though we

451 offer a simple linear equation for estimation of leakage assuming a liquid nitrogen control from leakage

452 data collected using a boiling control (Fig. 9), we suggest some caution in applying this conversion

453 widely, especially outside of the maple genus or to non-woody samples. Application to other systems may

454 benefit from experimental determination of a linear equation relevant to the samples in question.

456 Finally, we did not expect to find the diversity in maximum freezing-induced electrolyte leakage that we

457 found among species in our own and in re-analyzed studies (Additional file 2). Though this value appears

458 to depend somewhat on the lineage being sampled and on the time of year, it is not clear what underlying

459 mechanism causes such variety in the difference between electrolyte leakage following deep freezing and

460 boiling among woody samples. Future work might explore a connection between this maximum freezing-

461 induced leakage and cold hardiness.

\section{Methods}

464 Study System

465 The roughly 120 species of the maple genus (Acer L.) are distributed widely throughout the northern

466 hemisphere, having diverged from relatives in the Sapindaceae some 60 million years ago and radiated

467 from their center of diversity in eastern China to mesic environments throughout Asia, Europe, and North

468 America (Fig. 2; [38]). The maples are a typical temperate, woody genus, including common forest

469 dominants and rare and subordinate species, with species' native distributions covering areas with mild 
470 winters (Taiwan, Mexico) through very cold ones (Scandinavia, the Amur Valley). In the present study,

471 we focus on twelve maple species spanning the maple phylogeny $[39,40]$ and three principle

472 biogeographic realms colonized by the genus: Asia, North America, and Europe (Fig. 2; Grossman in

473 review).

474

475 All plant material was collected from mature, healthy trees at the Arnold Arboretum $\left(42^{\circ} 18^{\prime} 26^{\prime \prime} \mathrm{N}\right.$,

$47671^{\circ} 07^{\prime} 13 \mathrm{~W}$ ', $15-79 \mathrm{~m}$ a.s.1) in Boston, MA, USA. The Arboretum has a hot summer continental climate

477 ( $c f a$ in the Köppen-Geiger system) with a historical mean annual temperature of $9.7^{\circ} \mathrm{C}$ and mean annual

478 precipitation of $1168 \mathrm{~mm}$. Collections were primarily from accessioned trees in the Arnold's living

479 collections and occasionally from spontaneous individuals growing on the Arboretum grounds.

480 Information about all sampled trees is provided in Additional file 6.

482 We obtained previously published electrolyte leakage data for six southwestern U.S. oak species

483 (Quercus spp.; [11]). We also extracted some data from supplements associated with Kreyling and

484 colleagues' [10] study of diverse woody species at the Ecological Botanical Garden in Bayreuth,

485 Germany.

486

$487 \quad$ Electrolyte Leakage Measurements

488 We developed our electrolyte leakage routine by drawing on published accounts and testing a variety of

489 alternative methods. First, we collected material from three genotypes per species $(n=36)$, completing all

490 sampling on 13 March 2020, noting the phenological stage of each sampled individual. Healthy, one to

491 two year-old stems, typically $3-10 \mathrm{~mm}$ in diameter and $<30 \mathrm{~cm}$ in length were harvested and placed in

492 sealed plastic bags to prevent desiccation. These were then stored at $4{ }^{\circ} \mathrm{C}$ to prevent further

493 de/acclimation until sample preparation and testing. Processing consisted of cutting internode stems into

$49410 \mathrm{~mm}$ segments and sealing them in $15 \mathrm{~mL}$ plastic test tubes with $2 \mathrm{~mL}$ of nanopure water $(<18.2 \mathrm{M} \Omega)$.

495 For each genotype, 21 segments were prepared. Allocated across 6 freezing temperatures and a control, 
these yielded three measurement replicates per genotype. Freezing trials then occurred either 12 or 36

497 hours after sample preparation was completed; samples were randomized across either the first or second

498 day of trials and stored at $4{ }^{\circ} \mathrm{C}$ prior to freezing.

500 Freezing trials occurred in a Tenney Environmental (New Columbia, PA, USA) Test Chamber with 501 thermocouple monitoring of temperature using an RDXL4SD data logger (Omega Engineering, Inc.,

502 USA). Target temperatures for freezing were: $4{ }^{\circ} \mathrm{C}$ (controls kept in a growth chamber); -10, -20, $-30,-40$, 503 and $-60{ }^{\circ} \mathrm{C}$ (in the Tenney Chamber); and $-80{ }^{\circ} \mathrm{C}$ (in a separate freezer). Samples in the Tenney Chamber 504 were initially cooled from a storage temperature of 4 to $0{ }^{\circ} \mathrm{C}$ over thirty minutes and then held at $0{ }^{\circ} \mathrm{C}$ for 505 an additional thirty minutes. They were then stepped down to $-10{ }^{\circ} \mathrm{C}$ at a rate of $-0.33{ }^{\circ} \mathrm{C} / \mathrm{min}$ and held for 506 one hour at the target temperature. This routine was repeated for $-20,-30,-40$, and $-60{ }^{\circ} \mathrm{C}$ with the rate of 507 temperature change between -40 and $-60{ }^{\circ} \mathrm{C}$ twice that of the rate between other steps. After one hour at $50860{ }^{\circ} \mathrm{C}$, samples assigned to a $-80{ }^{\circ} \mathrm{C}$ target were moved to a separate deep freezer and held there for one to 509 two hours. Samples were removed from the Tenney Chamber or deep freezer and allowed to return to 510 room temperature on the benchtop after being held at their target temperature. Visual inspection showed

511 that water in all tubes froze completely without exogenous ice seeding or inclusion of steel shot to 512 promote ice formation within the first freezing step $\left(-10^{\circ} \mathrm{C}\right)$. Thermocouple readings indicate that actual 513 sample temperature was on average $1.5^{\circ} \mathrm{C}$ colder than the target temperature; we use actual temperature 514 reached (e.g., -10.5 in lieu of $-10{ }^{\circ} \mathrm{C}$ ) for analysis.

516 Following sample thawing, $8 \mathrm{~mL}$ of nanopure water were added to each test tube and tubes were shaken 517 at $100 \mathrm{rpm}$ and room temperature for 16 hours. Conductivity of solution in each tube was then read with a 518 conductivity probe calibrated with a low-concentration $(0-200 \mu \mathrm{S} / \mathrm{cm})$ standard (Vernier Software and 519 Technology, Beaverton, OR, USA). Samples were hand-shaken immediately before conductivity 520 measurements; we found that this was essential for accurate readings. 
523 Out of concern with the realism and consistency of the current set of methods used in electrolyte leakage

524 studies, we have developed and employed here an alternative approach, immersing all sample tubes in

525 liquid nitrogen (at least $-200{ }^{\circ} \mathrm{C}$ as indicated by thermocouple readings) for thirty minutes following

526 initial conductivity readings. Samples were then allowed to thaw on the bench and shaken at room

527 temperature and $100 \mathrm{rpm}$ for 16 hours. Final conductivity measurements were then collected as described 528 above.

530 To validate the use of this new control, a separate set of stem samples $(n=176)$ from all 12 study species 531 collected on 3 and 10 February 2020 was used to carry out the protocol described above. Following 532 freezing in liquid nitrogen, we measured their conductivity, then autoclaved them at $120^{\circ} \mathrm{C}$ for thirty

533 minutes, loosening tube caps prior to autoclaving to reduce evaporation while preventing

534 overpressurization of tubes. (We autoclaved them at high heat to maximize electrolyte leakage.) We then

535 agitated tubes for 16 hours and measured conductivity, both as described above, allowing for comparison

536 between leakage following liquid nitrogen immersion and boiling.

538 Previous studies have suggested that frozen samples may not release all diffusible electrolytes for up to a 539 week following freezing and that samples exposed to autoclaving for short periods of time (e.g., 15 min.)

540 will continue to leak electrolytes for ten days or more [13a]. In order to ascertain that our incubation

541 timing (both post-freezing and post-autoclaving or liquid nitrogen immersion) and autoclave intensities

542 were sufficient to capture electrolyte diffusion, we carried out an additional experiment on only two

543 species, $A$. caudatifolium and A. campestre. These two maples represented the two extremes of cold

544 hardiness in our main experiments (Table 1), occupy distinct habitats (Fig. 2), and belong to

545 phylogenetically distinct sections [40]. For this additional experiment, we collected stem sections of one

546 genotype of each species (with three measurement replicates per genotype collected) on 29 December

5472020 and measured using the same electrolyte leakage protocol described above while varying the amount 
548 of time samples incubated after boiling and damage controls (both autoclaving and liquid nitrogen)

549 treatments and the control treatment applied. As such, conductivity of stem segments of both species was

550 measured $1,2,5$, or 7 days post-freezing and then exposed to either boiling at $100^{\circ} \mathrm{C}$ in the Tenney

551 Chamber, autoclaving at $120^{\circ} \mathrm{C}$, or liquid nitrogen immersion $(\mathrm{N}=504)$. Segments were then allowed to

552 incubate for the same length of time as their post-freezing incubation and conductivity was re-measured.

553 This experiment crossed incubation time (one day through one week) with control method (moderate

554 boiling, intense boiling, or liquid nitrogen) for two species expected to vary in their cold hardiness,

555 producing 24 factor-level combinations.

$557 \quad$ Validation of Electrolyte Leakage

558 To validate estimates of cold hardiness from electrolyte leakage, we performed two additional cold

559 hardiness assays on stem tissue from sampled individuals: differential thermal analysis and visual

560 inspection of freezing damage. We briefly describe these procedures below.

561

562 Differential thermal analysis. Differential thermal analysis (DTAs; [41-43]) involves cooling plant

563 tissue of interest to progressively lower temperatures while using thermocouples to detect the tissue's

564 release of heat associated with the freezing of water, an exothermic reaction. Extracellular water in stems,

565 buds, etc. freezes at relatively warm sub-zero temperatures, producing a release of heat or high-

566 temperature exotherm (HTE); the freezing of this extracellular water is not considered harmful to

567 acclimated, cold-adapted woody plants. Intracellular water, on the other hand, will generally supercool to

568 much lower temperatures, reaching a limit at about $-42{ }^{\circ} \mathrm{C}$. Freezing of this supercooled water, which is

569 associated with catastrophic damage to affected cells, results in a low-temperature exotherm (LTE),

570 which can be detected through DTA and compared to results from electrolyte leakage assays $[8,14,16,24]$.

571

572 For DTA measurements, carried out on 16 March 2020, we used the same material collected for our 573 electrolyte leakage trials; this material had been stored with proximal ends inserted in water, at $2{ }^{\circ} \mathrm{C}$ for 
574 the intervening time ( 3 days). For each genotype ( $\mathrm{n}=36)$, we cut ten $30 \mathrm{~mm}$ segments of the same

575 internodal stem tissue and pooled these measurement replicates into a cell in which they were exposed to

576 Peltier plates, which can detect exotherms and convert their thermal signals to voltage. Samples were then

577 cooled at a rate of $-4{ }^{\circ} \mathrm{C} /$ hour to $-60^{\circ} \mathrm{C}$. Exotherm-associated voltages were collected using a Keithley

578 Multimeter Data Acquisition System (Keithley Instruments, Cleveland, OH, USA). HTE peaks were

579 discarded and LTE peaks were manually curated in Microsoft Excel prior to statistical analysis.

581 Visual damage inspection. We also visually inspected damage to cortical tissues cells following

582 freezing; this metric serves as an intuitive and holistic, though labor-intensive method for assessing cold

583 hardiness $[8,10,14,19,44]$. On 16 March 2020, we prepared samples for freezing followed by visual

584 damage inspection from the same material used in other trials. For each genotype $(n=36)$, and each

585 temperature treatment, we cut three $30 \mathrm{~mm}$ segments of internodal stem tissue and incubated them in

586 plastic scintillation vials with $2 \mathrm{~mL}$ water at $2{ }^{\circ} \mathrm{C}$ overnight. On $17 \mathrm{March}$, we froze these vials using the

587 same freezing routine as the one employed in our electrolyte leakage measurements. One set of vials was

588 kept at $4{ }^{\circ} \mathrm{C}$. The others were frozen to $-10,-20,-30,-40$, and $-80^{\circ} \mathrm{C}$. After removal from the freezer, vials

589 were allowed to thaw at $4{ }^{\circ} \mathrm{C}$ for 24 hours, then incubated with ends in water, at room temperature for six 590 days. At this time, they were evaluated using a quartile $(0 \%, 25 \%, 50 \%, 75 \%$ or $100 \%$ brown) damage 591 scale.

593 Data Analysis

594 All analyses were carried out in R (ver. 3.6.3, [45]).

595

596 Treatment of raw electrolyte leakage data. Raw electrolyte leakage at each temperature was

597 normalized into a relative electrolyte leakage $\left(R_{T}\right)$ at temperature $T$ :

598

599

$$
R_{T}=L_{T} / L_{k}
$$


601 where $L_{T}$ is the conductivity measured from a sample frozen at $T$ and $L_{k}$ is the conductivity of the same 602 sample after boiling. $R_{T}$ is the measurement used for damage estimation based on the Kreyling et al. [10] 603 approach. We then converted $R_{T}$ to Index of Injury $\left(I_{T}\right)$ for any given temperature $T$ following Flint and 604 colleagues' [7] method such that:

605

606

$$
I_{T}=100 \times \frac{\left(R_{T}-R_{o}\right)}{\left(1-R_{o}\right)}
$$

609 Where $R_{o}=R_{T}$ for an unfrozen control. In our case, the unfrozen control was kept at $4{ }^{\circ} \mathrm{C}$. Therefore, $I_{T}=$ 6100 at $T=4{ }^{\circ} \mathrm{C}$.

611

612 The third method employed was a modified version of Lim and colleagues' [12]. This adjustment builds

613 upon the apparent plateau observed in levels of damage in lower temperatures and assumes maximum

614 damage occurred within the temperatures tested:

615

616

$$
I_{T, a d j}=100 \times I_{T} / I_{\max } \equiv 100 \times \frac{\left(R_{T}-R_{o}\right)}{\left(R_{\max }-R_{o}\right)}
$$

618 where $I_{\max }$ and $R_{\max }$ are the maximum unadjusted injury and relative conductivity measured for any 619 genotype (usually attained at either -60 or $-80^{\circ} \mathrm{C}$ ).

620

621 Analysis of electrolyte leakage and visual damage data. Electrolyte leakage values of $R_{T}, I_{T}$, and $I_{T, \text { adj }}$, 622 and visual damage $(V D)$ were then modeled for each genotype using a four-parameter log-logistic model: 


$$
R_{T}, I_{T}, I_{a d j, T}, V D_{T}=c+\frac{d-c}{1+e^{(b \times((T)-(u)))}}
$$

626 where $T$ is the temperature, $e$ is euler's number, and $d, c, b$, and $u$ are the parameters estimated: $c$ is the 627 lower limit $\left(c=0\right.$ for $I_{T}, I_{a d j, T}$, and $\left.V D_{T}\right) ; d$ is the upper limit $\left(d=100\right.$ for $I_{a d j, T}$ and $\left.V D_{T}\right) ; b$ is the slope 628 associated with the logistic function; and $u$ is the inflection point. Here $L T_{50}=u$. For $I_{T, \text { adj }}$ we also 629 extracted values of $L T_{20}$ and $L T_{80}$ for comparison with $L T_{50}$. (Additional file 5).

630

631 We also modeled $I_{a d j, T}$ using a Gompertz function and extract both $L T_{50}$ and $L T_{\max }$ as a critical value of 632 cold hardiness as follows:

$$
I_{a d j, T}=100 \times e^{-b \times e^{-k \times T}}
$$

$$
L T_{\max }=\frac{-\log (1 / b)}{k}
$$

638 where $T$ is freezing temperature, $e$ is euler's number, and $b$ and $k$ are parameters. We fit Gompertz curves

639 for each genotype in our dataset using the $n l s$ function, whereas log-logistic functions were modeled using 640 the $d r m$ funditon in the drc package [46].

642 Critical values of $L T_{50}$ were extracted from the curves obtained from each genotype for each model for

643 comparison of means at a species level. For $I_{a d j}$, values of $L T_{20}$ and $L T_{80}$ were also used. We also

644 calculated the lowest survival temperature (LST) on a species basis for visual damage, which we defined

645 as the lowest temperature at which no stem segment experienced more than $50 \%$ damage $[8,41]$. Species

646 differences for these were analyzed using simple linear regression models and Type I ANOVAs. Tukey's 
648 significant difference.

650 Bivariate correlation tests were used to compare values of $L T_{20}, L T_{50}$ and $L T_{80}$ for the three electrolyte

651 leakage models with $L T_{20}, L T_{50}$ and $L T_{80}$ and LST values for visual damage. Further comparisons in terms

652 of correlation, bias, and RMSE were made from $L T_{10}$ to $L T_{90}$ in $10 \%$ steps for $I_{a d j}$ and $V D$.

654 Using raw electrolyte leakage data from a different study comparing acclimated and unacclimated oaks

655 [11], we followed the same protocol described to obtain $R, I$ and $I_{a d j}$. Since there were multiple genotypes 656 within each species, but no repetition within temperatures for each genotype, the control level used for $I$ 657 and $I_{a d j}$ was the lowest electrolyte leakage at either $4{ }^{\circ} \mathrm{C}$ or $-5{ }^{\circ} \mathrm{C}$ within each genotype. The three forms of 658 leakage data were used for log-logistic curve estimations of $T_{50}$, whereas $I_{a d j}$ was used for $T_{50}$ and $T_{\max }$ 659 estimations based on the Gompertz function as well.

660

661 Comparison of liquid nitrogen and boiling leakage. To compare the values of electrolyte leakage in

662 both types of control, Deming (or "least rectangle") regressions were fit. This type of regression takes into 663 account errors in both axes to find the best fit line. Although we did not include a pure water sample 664 control in the measurements, initial fitting showed a non-significant intercept, and therefore further fitting 665 was done with a zero-intercept model. Regular least-squares $\mathrm{R}^{2}$ values were calculated in both directions 666 to evaluate fitness of the model, from which a linear allometric relationship was extracted.

668 Damage estimated based on electrolyte leakage standardized on the liquid nitrogen control was then 669 calculated and fit based on $R$ and $I$ approaches. Fitted values were then compared between values of $I_{a d j}-$ 670 for which the control method has no influence - and $R$ and $I$ for both boiling and liquid nitrogen control. 
672 To understand how leakage following boiling compares to that caused by deep freezing across study

673 systems, we compared our maple data to leakage measurements taken on diverse species in two other

674 studies. We extracted additional $R$ data at liquid nitrogen temperatures from figures in the supplementary

675 data of Kreyling et al. [10] using a grid with 5\% increments. This dataset contained electrolyte leakage for

67627 species, 3 genotypes per species, at 3 different points: November, February, and March. Species and

677 time differences were analyzed using simple linear regression models and Type I ANOVAs. Tukey’s

678 HSD post-hoc tests were used to discriminate among groups. We also used data from the lowest

679 temperature used by Fallon and Cavender-Bares [11] for similar analyses using species and time

680 (acclimated vs. unacclimated) as variables. Although the Deming regressions used in our data are more

681 appropriate, we used $R$ values at liquid nitrogen temperatures and analyzed it using linear regression with

682 species as the explanatory variable for comparison with the other studies.

683

684 Impacts of incubation time and control method on electrolyte diffusion. To assess whether electrolyte

685 leakage depended on species identity, control methodology, or incubation time, we built two fixed-effects

686 linear models (using $\mathrm{lm}$ ) of electrolyte leakage measured in our focused study of $A$. caudatifolium and $A$.

687 campestre stem segments. These models both took the form of:

$$
L_{T}, L_{K} \sim \beta_{\text {Intercept }}+\beta_{\text {Temperature }}+\beta_{\text {Species }} * \beta_{\text {Incubation }} * \beta_{\text {Control }}+\varepsilon
$$

690 In this analysis, either leakage following freezing $\left(L_{T}\right)$ or exposure to boiling or liquid nitrogen $\left(L_{K}\right)$ was 691 modeled as a linear function of species identity, incubation time (a continuous variable consisting of 1, 2, 6925 , or 7 days), and control type (autoclaving at $100^{\circ} \mathrm{C}$, autoclaving at $120^{\circ} \mathrm{C}$, or liquid nitrogen immersion). 693 Temperature of freezing treatment $\left(4^{\circ} \mathrm{C}\right.$ to $\left.-80^{\circ} \mathrm{C}\right)$ was included as a covariate as it is expected to affect 694 sample conductivity. Models were fit with all interactions except those with freezing temperature and 695 analyzed using a conservative Type III ANOVA. Differences among factor-level combinations were 696 assessed using Tukey tests, as described above. For further comparison of the impacts of differences in 
697 incubation time and control type on estimates of cold hardiness, critical values $\left(T_{50}\right)$ were extracted for

698 each factor-level combination using the Lim logistic $_{\text {approach. }}$

699

700 Declarations

701 Ethics approval and consent to participate

702 Not applicable

703

704 Consent for publication

705 Not applicable

706

707 Availability of data and materials

708 All data generated by the authors during this study (on Acer samples) are included in this published article

709 and its supplementary information files. Re-analyzed data provided from other authors are available from

710 the corresponding author on reasonable request and pending permission from the originating author(s).

711 Code and maple data are also available at https://github.com/apkovaleski/EL_Methods.

712

713 Competing interests

714 The authors declare that they have no competing interests.

715

$716 \quad$ Funding

717 Both authors were supported by Katharine H. Putnam Fellowships in Plant Science administered by the

718 Arnold Arboretum of Harvard University.

719

720 Authors' contributions 
721 APK and JJG jointly and equally conceived of the original project idea, designed the experiment,

722 performed all field and laboratory work, and analyzed resultant data. Both authors contributed to all drafts

723 of the manuscript.

724

725 Acknowledgements

726 The authors wish to acknowledge support of the Katharine H. Putnam Fellowship in Plant Science and

727 thank Beth Fallon, Jeannine Cavender-Bares, Leena Lindén, and Pauliina Palonen for providing their own

728 data for re-analysis. Faye Rosin and Amelia Keyser-Gibson provided critical support in the execution of

729 this research.

730

731

732

733

734 


\section{Tables}

Table 1. Phenological condition and cold hardiness of A) maple and B) oak species.

\begin{tabular}{|c|c|c|c|c|c|c|c|c|c|}
\hline \multirow{3}{*}{ Species } & \multirow{3}{*}{ Condition } & \multicolumn{5}{|c|}{ Critical Values for Electrolyte Leakage } & \multirow{3}{*}{$\begin{array}{l}\text { Visual LTso, } \\
{ }^{\circ} \mathrm{C}\end{array}$} & \multirow{3}{*}{ LST, ${ }^{\circ} \mathrm{C}$} & \multirow{3}{*}{ LTE, ${ }^{\circ} \mathrm{C}$} \\
\hline & & Kreyling & Flint & Limlogistic & Limgompertz & Limgompertz & & & \\
\hline & & $\mathrm{LT}_{50},{ }^{\circ} \mathrm{C}$ & $\mathrm{LT}_{50},{ }^{\circ} \mathrm{C}$ & $\mathrm{LT}_{50},{ }^{\circ} \mathrm{C}$ & $\mathrm{LT}_{50},{ }^{\circ} \mathrm{C}$ & $\mathrm{LT}_{\max },{ }^{\circ} \mathrm{C}$ & & & \\
\hline \multicolumn{10}{|c|}{ A. Maple (Acer) data from the present study } \\
\hline A. saccharum & Dormant & $-28^{b}$ & $-28^{b}$ & $-26^{\mathrm{cd}}$ & $-26^{\mathrm{cd}}$ & $-21^{\mathrm{bc}}$ & $-29^{\mathrm{ab}}$ & $-33^{\mathrm{ab}}$ & $-36^{\mathrm{bc}}$ \\
\hline A. pseudoplatanus & Dormant & $-20^{\mathrm{ab}}$ & $-20^{\mathrm{ab}}$ & $-22^{\mathrm{bc}}$ & $-22^{\text {bcd }}$ & $-18^{\mathrm{abc}}$ & $-30^{\mathrm{ab}}$ & $-33^{\mathrm{ab}}$ & $-38^{\mathrm{cd}}$ \\
\hline A. rubrum & Budbreak & $-16^{\mathrm{ab}}$ & $-16^{\mathrm{ab}}$ & $-17^{\mathrm{ab}}$ & $-17^{\mathrm{ab}}$ & $-14^{\mathrm{ab}}$ & $-18^{\mathrm{a}}$ & $-17^{\mathrm{a}}$ & $-35^{b}$ \\
\hline A. platanoides & Dormant & $-25^{\mathrm{ab}}$ & $-24^{\mathrm{ab}}$ & $-25^{\mathrm{bcd}}$ & $-25^{\mathrm{bcd}}$ & $-20^{b c}$ & $-27^{a b}$ & $-30^{\mathrm{ab}}$ & $-35^{b}$ \\
\hline A. campestre & Dormant & $-25^{\mathrm{ab}}$ & $-25^{b}$ & $-32^{\mathrm{d}}$ & $-33^{\mathrm{d}}$ & $-26^{c}$ & $-36^{b}$ & $-40^{\mathrm{b}}$ & $-37^{\mathrm{cd}}$ \\
\hline A. hyrcanum & Dormant & $-23^{b}$ & $-23^{\mathrm{ab}}$ & $-23^{\mathrm{bc}}$ & $-23^{\mathrm{bcd}}$ & $-18^{a b c}$ & $-30^{\mathrm{ab}}$ & $-33^{\mathrm{ab}}$ & $-36^{\mathrm{ab}}$ \\
\hline A. tataricum & Bud swelling & $-18^{\mathrm{ab}}$ & $-18^{\mathrm{ab}}$ & $-19^{a b c}$ & $-19^{a b c}$ & $-14^{\mathrm{ab}}$ & $-19^{a}$ & $-20^{\mathrm{ab}}$ & $-36^{\text {bcd }}$ \\
\hline A. tegmentosum & Budbreak & $-18^{\mathrm{ab}}$ & $-17^{\mathrm{ab}}$ & $-18^{\mathrm{abc}}$ & $-18^{\mathrm{abc}}$ & $-14^{\mathrm{ab}}$ & $-17^{a}$ & $-23^{\mathrm{ab}}$ & $-36^{\mathrm{bcd}}$ \\
\hline A. caudatifolium & Budbreak & $-12^{\mathrm{a}}$ & $-12^{\mathrm{a}}$ & $-13^{\mathrm{a}}$ & $-13^{\mathrm{a}}$ & $-11^{\mathrm{a}}$ & $-20^{\mathrm{a}}$ & $-20^{\mathrm{ab}}$ & $-20^{\mathrm{a}}$ \\
\hline A. davidii & Bud swelling & $-17^{\mathrm{ab}}$ & $-18^{\mathrm{ab}}$ & $-19^{a b c}$ & $-20^{\mathrm{abc}}$ & $-16^{\mathrm{ab}}$ & $-21^{\mathrm{a}}$ & $-27^{a b}$ & $-20^{\mathrm{a}}$ \\
\hline A. spicatum & Dormant & $-20^{\mathrm{ab}}$ & $-21^{\mathrm{ab}}$ & $-20^{\mathrm{abc}}$ & $-21^{a b c}$ & $-16^{a b}$ & $-27^{a b}$ & $-23^{\mathrm{ab}}$ & $-38^{\mathrm{d}}$ \\
\hline A. negundo & Dormant & $-27^{b}$ & $-27^{b}$ & $-24^{\mathrm{bcd}}$ & $-26^{\mathrm{bcd}}$ & $-18^{\mathrm{abc}}$ & $-20^{\mathrm{a}}$ & $-23^{\mathrm{ab}}$ & $-36^{\mathrm{bcd}}$ \\
\hline \multicolumn{7}{|c|}{ B. Oak (Quercus) data from Fallon and Cavender-Bares (2018) } & \multicolumn{2}{|c|}{ Published $\mathrm{LT}_{50},{ }^{\circ} \mathrm{C}$} & $\mathbf{N}$ \\
\hline \multirow{2}{*}{ Q. arizonica } & Acclimated & $-22^{\mathrm{b}}$ & $-21^{\mathrm{bc}}$ & $-21^{\mathrm{bc}}$ & $-21^{\mathrm{bc}}$ & $-18^{\mathrm{b}}$ & \multicolumn{2}{|c|}{-34} & 39 \\
\hline & Unacclimated & $-12^{\mathrm{a}}$ & $-12^{\mathrm{a}}$ & $-12^{\mathrm{a}}$ & $-12^{\mathrm{a}}$ & $-10^{\mathrm{a}}$ & \multicolumn{2}{|c|}{-16} & 44 \\
\hline \multirow{2}{*}{ Q. emoryi } & Acclimated & $-26^{c}$ & $-26^{\mathrm{d}}$ & $-25^{\mathrm{d}}$ & $-26^{\mathrm{d}}$ & $-23^{c}$ & \multicolumn{2}{|c|}{-29} & 12 \\
\hline & Unacclimated & $-14^{\mathrm{a}}$ & $-14^{\mathrm{a}}$ & $-14^{\mathrm{a}}$ & $-14^{\mathrm{a}}$ & $-11^{\mathrm{a}}$ & \multicolumn{2}{|c|}{-15} & 10 \\
\hline \multirow{2}{*}{ Q. gambelii } & Acclimated & $-22^{\mathrm{b}}$ & $-21^{b c}$ & $-21^{b c}$ & $-21^{b c}$ & $-18^{\mathrm{b}}$ & \multicolumn{2}{|c|}{-33} & 17 \\
\hline & Unacclimated & $-11^{\mathrm{a}}$ & $-11^{a}$ & $-12^{a}$ & $-12^{\mathrm{a}}$ & $-10^{a}$ & \multicolumn{2}{|c|}{-20} & 16 \\
\hline \multirow{2}{*}{ Q. grisea } & Acclimated & $-25^{b c}$ & $-25^{\mathrm{cd}}$ & $-25^{\mathrm{cd}}$ & $-26^{\mathrm{cd}}$ & $-23^{c}$ & \multicolumn{2}{|c|}{ Not calculated } & 2 \\
\hline & Unacclimated & $-16^{\mathrm{ab}}$ & $-15^{\mathrm{ab}}$ & $-15^{\mathrm{ab}}$ & $-15^{\mathrm{ab}}$ & $-12^{\mathrm{ab}}$ & \multicolumn{2}{|c|}{ Not calculated } & 2 \\
\hline \multirow{2}{*}{ Q. hypoleucoides } & Acclimated & $-22^{b}$ & $-21^{b c}$ & $-21^{b c}$ & $-21^{b c}$ & $-18^{b}$ & \multicolumn{2}{|c|}{-23} & 39 \\
\hline & Unacclimated & $-13^{\mathrm{a}}$ & $-13^{\mathrm{a}}$ & $-13^{\mathrm{a}}$ & $-13^{\mathrm{a}}$ & $-11^{\mathrm{a}}$ & \multicolumn{2}{|c|}{-13} & 40 \\
\hline \multirow{2}{*}{ Q. rugosa } & Acclimated & $-24^{\mathrm{bc}}$ & $-23^{\mathrm{cd}}$ & $-23^{\mathrm{cd}}$ & $-23^{\mathrm{cd}}$ & $-19^{b c}$ & \multicolumn{2}{|c|}{-32} & 16 \\
\hline & Unacclimated & $-13^{\mathrm{a}}$ & $-13^{\mathrm{a}}$ & $-13^{\mathrm{a}}$ & $-13^{a}$ & $-11^{\mathrm{a}}$ & \multicolumn{2}{|c|}{-17} & 17 \\
\hline
\end{tabular}

Phenological condition at sampling and critical values of cold hardiness for electrolyte leakage, lowest survival temperatures (LSTs), and low temperature exotherms (LTEs) for A) maple species $(\mathrm{n}=3)$ and $\mathrm{B}$ ) oak species-acclimation combinations (sample size and originally published critical values as noted; Fallon and Cavender-Bares, 2018). In cases for which differences among species are significant, superscripts indicate results of a Tukey's HSD post-hoc test $(\alpha=0.05)$. Samples vary in phenological condition at the time of sampling. Maples were sampled at various points ranging from dormancy to post-budbreak in early spring. Oaks were sampled while either cold-acclimated (winter) or unaccclimated (summer). 
Table 2. Correlations between critical values of cold hardiness based on different approaches.

\begin{tabular}{|c|c|c|c|c|c|c|c|c|c|c|c|c|c|c|c|c|c|c|}
\hline & & \multicolumn{3}{|c|}{ Kreyling } & \multicolumn{3}{|c|}{ Flint } & \multicolumn{3}{|c|}{ Lim $_{\text {logistic }}$} & \multicolumn{4}{|c|}{ Lim $_{\text {gompertz }}$} & \multicolumn{4}{|c|}{ Visual Damage } \\
\hline & & $\mathrm{LT}_{20}$ & $\mathrm{LT}_{50}$ & $\mathrm{LT}_{80}$ & $\mathrm{LT}_{20}$ & $\mathrm{LT}_{50}$ & $\mathrm{LT}_{80}$ & $\mathrm{LT}_{20}$ & $\mathrm{LT}_{50}$ & $\mathrm{LT}_{80}$ & $\mathrm{LT}_{20}$ & $\mathrm{LT}_{50}$ & $\mathrm{LT}_{80}$ & $\mathrm{LT}_{\max }$ & $\mathrm{LT}_{20}$ & $\mathrm{LT}_{50}$ & $\mathrm{LT}_{80}$ & LST \\
\hline \multirow{4}{*}{ Kreyling } & $\mathrm{LT}_{20}$ & & & & & & & & & & & & & & & & & \\
\hline & $\mathrm{LT}_{50}$ & 0.44 & & & & & & & & & & & & & & & & \\
\hline & $\mathrm{LT}_{80}$ & -0.1 & 0.84 & & & & & & & & & & & & & & & \\
\hline & $\mathrm{LT}_{20}$ & 0.92 & 0.54 & 0.6 & & & & & & & & & & & & & & \\
\hline \multirow[t]{3}{*}{ Flint } & $\mathrm{LT}_{50}$ & 0.4 & Identical & 0.85 & 0.53 & & & & & & & & & & & & & \\
\hline & $\mathrm{LT}_{80}$ & -0.3 & 0.88 & 0.99 & 0.1 & 0.89 & & & & & & & & & & & & \\
\hline & $\mathrm{LT}_{20}$ & 0.89 & 0.6 & 0.11 & 0.94 & 0.59 & 0.17 & & & & & & & & & & & \\
\hline \multirow[t]{3}{*}{$\operatorname{Lim}_{\text {logistic }}$} & $\mathrm{LT}_{50}$ & 0.59 & 0.81 & 0.5 & 0.72 & 0.81 & 0.55 & 0.74 & & & & & & & & & & \\
\hline & $\mathrm{LT}_{80}$ & 0.2 & 0.7 & 0.63 & 0.34 & 0.72 & 0.65 & 0.33 & 0.88 & & & & & & & & & \\
\hline & $\mathrm{LT}_{20}$ & 0.91 & 0.48 & -0.2 & 0.98 & 0.47 & 0.3 & 0.96 & 0.69 & 0.29 & & & & & & & & \\
\hline \multirow{4}{*}{ Lim $_{\text {gompertz }}$} & $\mathrm{LT}_{50}$ & 0.52 & 0.82 & 0.56 & 0.65 & 0.83 & 0.6 & 0.68 & Identical & 0.91 & 0.62 & & & & & & & \\
\hline & $\mathrm{LT}_{80}$ & 0.24 & 0.78 & 0.68 & 0.37 & 0.8 & 0.72 & 0.41 & 0.91 & 0.98 & 0.33 & 0.94 & & & & & & \\
\hline & $L T_{\text {max }}$ & 0.7 & 0.78 & 0.41 & 0.83 & 0.78 & 0.46 & 0.84 & 0.98 & 0.78 & 0.81 & 0.96 & 0.82 & & & & & \\
\hline & $\mathrm{LT}_{20}$ & 0.28 & 0.28 & 0.13 & 0.34 & 0.3 & 0.16 & 0.35 & 0.42 & 0.34 & 0.34 & 0.4 & 0.34 & 0.42 & & & & \\
\hline \multirow{4}{*}{$\begin{array}{l}\text { Visual } \\
\text { Damage }\end{array}$} & $\mathrm{LT}_{50}$ & 0.44 & 0.46 & 0.22 & 0.5 & 0.47 & 0.27 & 0.51 & 0.6 & 0.49 & 0.49 & 0.58 & 0.5 & 0.61 & 0.85 & & & \\
\hline & $\mathrm{LT}_{80}$ & 0.46 & 0.49 & 0.24 & 0.51 & 0.5 & 0.3 & 0.53 & 0.62 & 0.49 & 0.5 & 0.6 & 0.51 & 0.62 & 0.58 & 0.92 & & \\
\hline & LST & 0.49 & 0.43 & 0.14 & 0.51 & 0.43 & 0.21 & 0.54 & 0.59 & 0.46 & 0.52 & 0.57 & 0.47 & 0.61 & 0.48 & 0.8 & 0.89 & \\
\hline & LTE & $\overline{0 .}$ & -0.64 & $\overline{0.58}$ & $\overline{0.31}$ & $\overline{0.63}$ & $\overline{0 .}$ & $\overline{0 .}$ & -0.46 & $\overline{0.45}$ & $0 . \overline{27}$ & $\overline{0.47}$ & $\overline{0.48}$ & -0.43 & $\overline{-} \overline{26}$ & $\overline{0 .}$ & $\overline{-} \overline{35}$ & $0 . \overline{28}$ \\
\hline
\end{tabular}

Correlations $(\rho)$ between critical values of cold hardiness $\left({ }^{\circ} \mathrm{C}\right)$ for four electrolyte leakage approaches, visual damage (including lowest survival temperature), and low temperature exotherms. Correlations are calculated for maple genotype means $(n=36)$ for all indices except LTEs, for which species means $(n=12)$ are compared to other metrics. We focus on the use of the LT50 critical value extracted using the Lim $_{\text {logistic }}$ approach (enclosed with borders). Bolded correlations indicate a significant $(\alpha<0.05)$ bivariate correlation test. 


\section{Figure Legends}

Figure 1. Schematic comparing different approaches to measuring electrolyte leakage. As described in the main text (Fig. 2), we contrast the Kreyling approach (no minimum or maximum leakage specified, yields $R$ ) with the Flint approach (minimum but no maximum, yields $I$ ) and the Lim approach (minimum and maximum specified, yields $I_{a d j}$ ). A) In the Kreyling approach, the temperature at which a sample accrues $50 \%$ of possible damage (T50 for $R$ ) may not be meaningful; many samples may not reach this "Absolute $50 \%$ " damage point. Instead, the temperature at which "Relative 50\%" damage is attained may be more meaningful. B) By comparison, values of $I$, in the Flint approach (orange line) are zeroed, although this may not drastically displace the curve relative to a Kreyling curve. C) In the Lim approach, data are forced to reach $100 \%$ damage, usually at the coldest temperature employed to freeze samples. Curve shapes may differ and critical temperatures (e.g., $\mathrm{T}_{50}$ ) corresponding to $I_{a d j}$ (extracted from the Lim curve) may or may not differ from those associated with $R$ (Kreyling curve). D) Use of a liquid nitrogen control is expected to elevate all leakage values, making, for instance, a Kreyling curve behave more like a Lim curve and improving generalizability among approaches.

Figure 2. A) Native and naturalized distributions of the 12 maple study species, which are distributed across the North American (red/pink), European (green/yellow), and Asian (blue/purple) extent of the genus (Grossman, in review). Star indicates the location of the Arnold Arboretum. B) Phylogenetic relatedness and ecological descriptions for the study species. Phylogeny and section designations adapted from $[39,40]$.

Figure 3. Comparison of four approaches for fitting curves to data representing the relationship between freezing damage and temperature in (A, C, E, G) A. caudatifolium and (B, D, F, H) A. campestre stem segments (plots for other species provided in Appendix 1). Curves fit to data on a per-genotype (red, blue, and green) and per-species (black curve) basis are fit in each case. Panels show curves fit following the approach of A, B) Kreyling and colleagues' (2015), C, D) Flint et al. (1987), and Lim et al. (1998). Approaches vary, as indicated, in their use of room-temperature (zeroing; $\mathrm{C}-\mathrm{H}$ ) and deep freezing (maximum damage; E-H) controls and reliance on general logistic (A-F) vs. Gompertz (G-H) curves.

Figure 4. A) Validation of critical values from four approaches to modeling electrolyte leakage (as in Fig. 1) against visual estimates of freezing damage. Critical values reflect either $20 \%, 50 \%$, or $80 \%$ electrolyte leakage (rows) or visual damage (columns). The rightmost column indicates lowest survival temperature (LST), the lowest temperature at which stems experienced $<50 \%$ damage. Pie wedge size and color indicate correlation. B) 50\% electrolyte leakage values using the Lim logistic approach (orange box) best predicted visual damage in the $40-60 \%$ damage range.

Figure 5. Damage, as reflected by electrolyte leakage (solid lines) and visual estimates (dashed lines), induced by freezing from -10 to $80^{\circ} \mathrm{C}$. Electrolyte leakage is calculated using the Limlogistic approach. Panels represent estimates of damage to particular genotypes. Color-coding indicates species as in Fig. 2; species are also presented in alphabetical order.

Figure 6. Visual cambial damage corresponded to critical cold hardiness estimated from electrolyte leakage data. Values of T50 given here (Table 1) are calculated using the Limlogistic approach. 
Representative stem samples following freezing are shown for A) Acer caudatifolium, B) A. davidii, C) A. hyrcanum, and D) A. negundo. Scale bar $=0.5 \mathrm{~cm}$.

Figure 7. Critical electrolyte leakage (estimated using the Lim logistic approach) best approximates 50\% visual damage when leakage is between 50 and $80 \%$. Bias, though, is lowest from 20 to $50 \%$ leakage. Color-coding indicates species as in Fig. 2. Error reflects variation among genotypes of a given species.

Figure 8. Fitness characteristics of the relationship between fitted values of electrolyte leakage and visual damage. Red contour delimits the area where: A) Correlation is greater than 0.55 ; B) $\mathrm{Bias}<\mathrm{abs}\left(5^{\circ} \mathrm{C}\right)$; and RMSE $<7^{\circ} \mathrm{C}$. Dashed rectangle delimits data used in Figure 7.

Figure 9. A) Sample conductivity following boiling predicts conductivity following immersion in liquid nitrogen across a range of values and for diverse species (color-coding indicates species as in Fig. 2.). Circled points are statistical outliers and lines indicate Deming regression error. $\mathrm{r}^{2}$ were calculated based on residuals in each direction. B) When a boiling standard is used, electrolyte leakage values derived using different curve-fitting procedures (e.g. Kreyling vs. Lim logistic $_{\text {) }}$ are not comparable above $\sim 25 \%$ leakage. C) However, use of a liquid nitrogen standard makes outputs of these two routines more comparable. Grey bar indicates a range of values within $15 \%$ of the $1: 1$ line.

Figure 10. When a boiling standard is used, electrolyte leakage values derived using different curvefitting procedures (e.g. Kreyling vs. Limlogistic vs. Flint approaches) are not comparable above $\sim 25 \%$ leakage (A vs. B). However, use of a liquid nitrogen standard makes outputs of these two routines more comparable (C vs. D). Grey bar indicates a range of values within $15 \%$ of the $1: 1$ line.

Figure 11. A) Electrolyte leakage increased gradually over seven days following experimental freezing (light blue), but not exposure to a boiling or liquid nitrogen control (turquoise), with no evidence of a significant difference when conductivity was measured over the first 48 hours after freezing. Lowercase letters indicate significant differences in conductivity measured at different time points at the 0.05 level based on models reported in Additional file 3 panels A (light blue, a-c) and B (turquoise, d-e). B) As a result of this pattern, estimates of critical values for cold hardiness $\left(\mathrm{T}_{50}\right)$ are consistent and reflect species differences when samples were incubated for one or two days, but not when they were incubated for 5 or 7 days (Additional file 4).

Figure 12. Stem segments incubated for longer than five days following control treatment (boiling or liquid nitrogen immersion) tended to deteriorate, showing evidence of microbial growth. 


\section{Additional files (Additional_files_1-6.xlsx)}

Additional file 1. Parameters, error (in parentheses), and pseudo-R2 for models of freezing damage as measured by A-D) electrolyte leakage or E) visual observation of cambial browning. Models are fit to A-C, E) a general logistic curve or D) a Gompertz curve as described in the text.

Additional file 2. Maximum freezing-induced leakage (\%) attained across several electrolyte leakage studies. Mean separations reflect outcomes of Tukey's HSD post-hoc tests $(\alpha=0.05)$. A) Data for maple (Acer spp.) species included in the present study. Percentages indicate leakage following immersion in liquid nitrogen divided by leakage following boiling. Superscripts reflect species differences among means. B) Data for oak (Quercus spp.) species from Fallon and Cavender-Bares' (2018) study. Percentages indicate leakage following freezing to $-40^{\circ} \mathrm{C}$ (the lowest freezing temperature used in the study) divided by leakage following boiling. Superscripts reflect differences among means of species-treatment combinations. C) Data for a variety of species assessed during three times of year extracted from supplementary figures from Kreyling et al. (2015). Percentages indicate leakage following immersion in liquid nitrogen divided by leakage following boiling. Two axes of mean separation are presented. Differences among species within the same sampling month are indicated by lowercase letters. Differences among sampling months for a single species are indicated by uppercase letters. Color coding (red to blue) highlights species and temporal patterns in maximum freezing-induced leakage in the Kreyling et al. data.

Additional file 3. Results from Type III ANOVA of linear models assessing the consequences of species (A. caudatifolium vs. A. campestre), control type (boiling at 120C, boiling at $125 \mathrm{C}$, or liquid nitrogen immersion), and incubation time (1, 2, 5, or 7 days post-treatment) on A) conductivity after experimental freezing and B) conductivity after control treatment. Temperature of experimental freezing was included as a covariate.Samples were collected on 29 Dec. 2020.

Additional file 4. Critical cold hardiness (T50, degrees C) estimated using the Limlogistic approach for two species, A) Acer caudatifolium and B) A. campestre with control type (boiling at 120C, boiling at $125 \mathrm{C}$, or liquid nitrogen immersion) and incubation time (1, 2, 5, or $7^{*}$ days post-treatment) varied. Samples were collected on 29 Dec. 2020.

Additional file 5. Species means of critical temperatures at which tissue accrued $20 \%$ and $80 \%$ damage as measured with electrolyte leakage and calculated using the Limlogistic approach. In cases for which differences among species are significant, superscripts indicate results of a Tukey's HSD post-hoc test $(\alpha=0.05)$.

Additional file 6. Description of all sampled plants. Genotypes of 10 species are all accessioned individuals in the Arnold Arboretum's living collections, as detailed below. Samples of A. negundo were collected from three spontaneously occurring plants found in the Arboretum's Bussey Brook Meadow. Samples of A. platanoides were similarly collected from three spontaneous individuals growing on the Arboretum's Weld Hill parcel. 


\section{Literature Cited}

1. Gusta L V., Wisniewski M. Understanding plant cold hardiness: An opinion. Physiol Plant. 2013;147:4-14.

2. Edwards EJ, Chatelet DS, Chen BC, Ong JY, Tagane S, Kanemitsu H, et al. Convergence, consilience, and the evolution of temperate deciduous forests. Am Nat. 2017;190:S87-104.

3. Koehler K, Center A, Cavender-Bares J. Evidence for a freezing tolerance-growth rate trade-off in the live oaks (Quercus series Virentes) across the tropical-temperate divide. New Phytol. 2012;193:73044.

4. Hawkins BA, Rueda M, Rangel TF, Field R, Diniz-Filho JAF. Community phylogenetics at the biogeographical scale: Cold tolerance, niche conservatism and the structure of North American forests. J Biogeogr. 2014;41:23-38.

5. Muffler L, Beierkuhnlein C, Aas G, Jentsch A, Schweiger AH, Zohner C, et al. Distribution ranges and spring phenology explain late frost sensitivity in 170 woody plants from the Northern Hemisphere. Glob Ecol Biogeogr. 2016;25:1061-71.

6. Dexter ST, Tottingham WE, Graber LF. Investigations of the hardiness of plants by measurement of electrical conductivity. Plant Physiol. 1932;7:63-78.

7. Flint HL, Boyce BR, Beattie DJ. Index of Injury—a Useful Expression of Freezing Injury To Plant Tissues As Determined By the Electrolytic Method. Can J Plant Sci. 1967;47:229-30.

8. Aniśko T, Lindstrom OM. Applying the Richards function in freezing tolerance determination with electrolyte and phenolic leakage techniques. Physiol Plant. 1995;95:281-7.

9. Lenz A, Hoch G, Vitasse Y, Körner C. European deciduous trees exhibit similar safety margins against damage by spring freeze events along elevational gradients. New Phytol. 2013;200:1166-75.

10. Kreyling J, Schmid S, Aas G. Cold tolerance of tree species is related to the climate of their native ranges. J Biogeogr. 2015;42:156-66.

11. Fallon B, Cavender-Bares J. Leaf-level trade-offs between drought avoidance and desiccation recovery drive elevation stratification in arid oaks. Ecosphere [Internet]. 2018;9:e02149. Available from: http://doi.wiley.com/10.1002/ecs2.2149

12. Lim CC, Arora R, Townsend EC. Comparing Gompertz and Richards Functions to estimate freezing injury in Rhododendron using electrolyte leakage. J Am Soc Hortic Sci. 1998;123:246-52.

13. Deans JD, Billington HL, Harvey FJ. Assessment of frost damage to leafless stem tissues of Quercus petraea: A reappraisal of the method of relative conductivity. Forestry. 1995;68:25-34.

14. Fiorino P, Mancuso S. Differential thermal analysis, supercooling and cell viability in organs of Olea europaea at subzero temperatures. Adv Hortic Sci. 2000;14:23-7.

15. Guàrdia M, Charrier G, Vilanova A, Savé R, Ameglio T, Aletà N. Genetics of frost hardiness in Juglans regia L. and relationship with growth and phenology. Tree Genet Genomes [Internet]. Tree Genetics \& Genomes; 2016;12. Available from: http://dx.doi.org/10.1007/s11295-016-1038-3

16. Wu D, Kukkonen S, Luoranen J, Pulkkinen P, Heinonen J, Pappinen A, et al. Influence of late autumn preconditioning temperature on frost hardiness of apple, blueberry and blackcurrant saplings. Sci Hortic (Amsterdam) [Internet]. Elsevier; 2019;258:108755. Available from: https://doi.org/10.1016/j.scienta.2019.108755

17. Ouyang L, Leus L, De Keyser E, Van Labeke MC. Seasonal changes in cold hardiness and carbohydrate metabolism in four garden rose cultivars. J Plant Physiol [Internet]. Elsevier; 2019;232:188-99. Available from: https://doi.org/10.1016/j.jplph.2018.12.001

18. Von Fircks HA, Verwijst T. Plant viability as a function of temperature stress: The Richards function applied to data from freezing tests of growing shoots. Plant Physiol. 1993;103:125-30.

19. Lindén L, Palonen $P$, Lindén M. Relating freeze-induced electrolyte leakage measurements to lethal temperature in red raspberry. J Am Soc Hortic Sci. 2000;125:429-35.

20. Lee JH, Yu DJ, Kim SJ, Choi D, Lee HJ. Intraspecies differences in cold hardiness, carbohydrate content and $\beta$-amylase gene expression of Vaccinium corymbosum during cold acclimation and deacclimation. Tree Physiol. 2012;32:1533-40. 
21. Yu DJ, Hwang JY, Chung SW, Oh HD, Yun SK, Lee HJ. Changes in cold hardiness and carbohydrate content in peach (Prunus persica) trunk bark and wood tissues during cold acclimation and deacclimation. Sci Hortic (Amsterdam) [Internet]. Elsevier B.V.; 2017;219:45-52. Available from: http://dx.doi.org/10.1016/j.scienta.2017.02.038

22. Stuart NW. Comparative cold hardiness of scion roots from fifty apple varieties. Proc Am Soc Hortic Sci. 1939;37:330-4.

23. Zhang YJ, Bucci SJ, Arias NS, Scholz FG, Hao GY, Cao KF, et al. Freezing resistance in Patagonian woody shrubs: the role of cell wall elasticity and stem vessel size. Tree Physiol. 2016;36:1007-18.

24. Arora R, Wisniewski ME, Scorza R. Cold acclimation in genetically related (Sibling) deciduous and evergreen peach (Prunus persica [L] Batsch): I. Seasonal changes in cold hardiness and polypeptides of bark and xylem tissues. Plant Physiol. 1992;99:1562-8.

25. Pagter M, Jensen CR, Petersen KK, Liu F, Arora R. Changes in carbohydrates, ABA and bark proteins during seasonal cold acclimation and deacclimation in Hydrangea species differing in cold hardiness. Physiol Plant. 2008;134:473-85.

26. Deacon NJ, Grossman JJ, Cavender-Bares J. Drought and freezing vulnerability of the isolated hybrid aspen Populus x smithii relative to its parental species , P . tremuloides and P . grandidentata. Ecol Evol. 2019;1-13.

27. Ouyang L, Leus L, Van Labeke MC. Three-year screening for cold hardiness of garden roses. Sci Hortic (Amsterdam) [Internet]. Elsevier; 2019;245:12-8. Available from: https://doi.org/10.1016/j.scienta.2018.10.003

28. Odlum KD, Blake TJ. A comparison of analytical approaches for assessing freezing damage in black spruce using electrolyte leakage methods. Can J Bot. 2008;74:952-8.

29. Repo T, Mononen K, Alvila L, Pakkanen TT, Hänninen H. Cold acclimation of pedunculate oak (Quercus robur L.) at its northernmost distribution range. Environ Exp Bot. 2008;63:59-70.

30. Hodge GR, Dvorak WS, Tighe ME. Comparisons between laboratory and field results of frost tolerance of pines from the southern USA and Mesoamerica planted as exotics. South For. 2012;74:7-17.

31. Cho W, Chandra R, Lee S, Han J, Lee S, Tsetsegmaa G, et al. Cold hardiness of 8 hybrid poplar clones for the introduction to arid and semi-arid areas. Plant Breed Biotechnol. 2020;8:11-8.

32. Zhang MIN, Willison JHM. An improved conductivity method for the measurement of frost hardiness. Can J Bot. 1987;65:710-5.

33. Burr KE, Hawkins CDB, L'Hirondelle SJ, Binder WD, George MF, Repo T. Methods for measuring cold hardiness of conifers. In: Bigras FJ, Colombo SJ, editors. Conifer cold hardiness. Dordrecht: Kluwer Academic Publishers; 2001. p. 369-401.

34. Palonen P, Lindén L. Dormancy, cold hardiness, dehardening, and rehardening in selected red raspberry cultivars. J Am Soc Hortic Sci. 1999;124:341-6.

35. Savage JA, Cavender-Bares J. Phenological cues drive an apparent trade-off between freezing tolerance and growth in the family Salicaceae. Ecology. 2013;94:1708-17.

36. Li H, Li Q, Xing L, Sun G, Zhao X. Comparison of cold hardiness evaluation of woody species by ELLT and TTCLT. HortScience. 2020;55:1228-1232.

37. Prásil I, Zámečník J. Time course of electrolyte leakage from various samples killed by frost, liquid nitrogen or boiling. Biol. Plant. 1990;32:77-80.

38. van Gelderen DM, de Jong PC, Oterdoom HJ. Maples of the World. Portland, OR: Timber Press; 1994.

39. Li J, Stukel M, Bussies P, Skinner K, Lemmon AR, Lemmon EM, et al. Maple phylogeny and biogeography inferred from phylogenomic data. J Syst Evol. 2019;57:594-606.

40. Areces-Berazain F, Hinsinger DD, Strijk JS. Genome-wide supermatrix analyses of maples (Acer, Sapindaceae) reveal recurring inter-continental migration, mass extinction, and rapid lineage divergence. Genomics [Internet]. Elsevier Inc.; 2021;113:681-92. Available from: https://doi.org/10.1016/j.ygeno.2021.01.014 
41. Quamme HA. Use of thermal analysis to measure freezing resistance of grape buds. Can J Plant Sci. 1986;66:945-52.

42. Mills LJ, Ferguson JC, Keller M. Cold-hardiness evaluation of grapevine buds and cane tissues. Am J Enol Vitic. 2006;57:194-200.

43. Londo JP, Kovaleski AP. Characterization of wild North American grapevine cold hardiness using differential thermal analysis. Am J Enol Vitic. 2017;68:203-12.

44. Sakai A. Freezing tolerance of evergreen and deciduous broad-leaved trees in Japan with reference to tree regions. Low Temp Sci Ser B, Biol Sci [Internet]. 1979;36:1-19. Available from: e:\%5CMy References\%5CBiogeography\%5CSakai 1979 Low temperature science.pdf

45. R Core Team. R: A language and environment for statistical computing [Internet]. Vienna, Austria: $\mathrm{R}$ Foundation for Statistical Computing; 2020. Available from: https://www.r-project.org/

46. Ritz C, Baty F, Streibig JC, Gerhard D. Dose-Response Analysis Using R. PLoS One. 2015;10:e0146021.

47. de Mendiburu F. agricolae: Statistical procedures for agricultural research [Internet]. 2020. Available from: https://cran.r-project.org/package=agricolae 

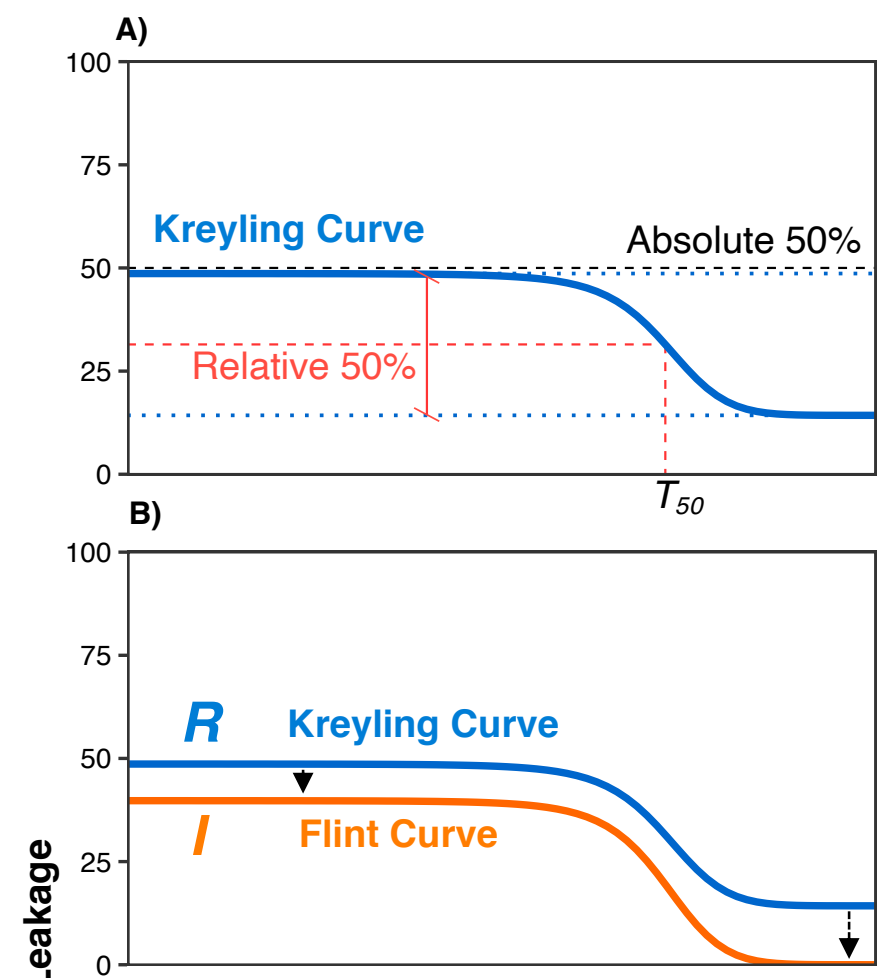

C)



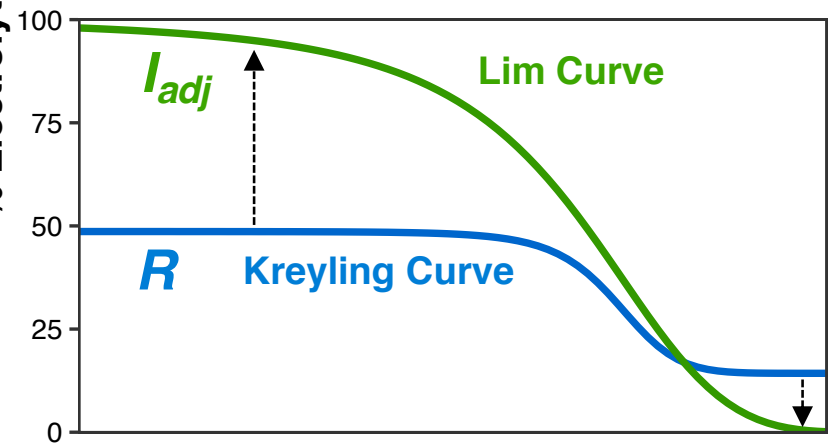

D)

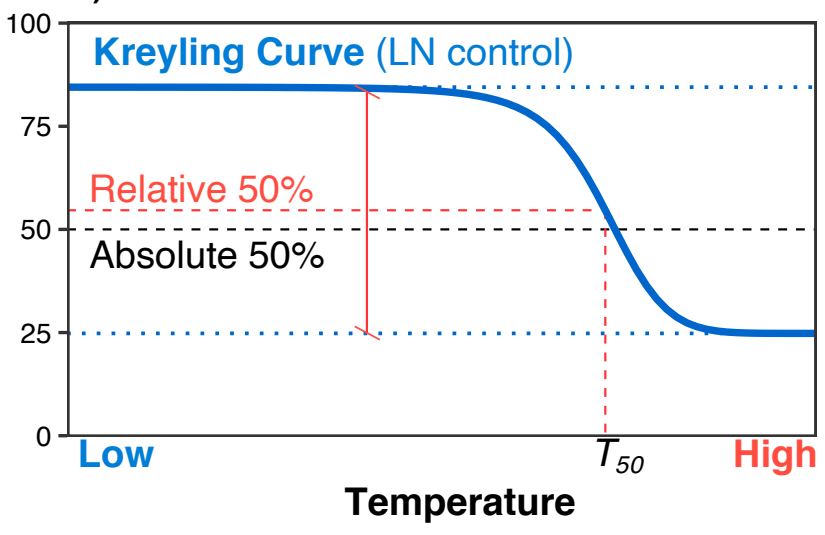

Figure 1. Schematic comparing different approaches to measuring electrolyte leakage. As described in the main text (Fig. 2), we contrast the Kreyling approach (no minimum or maximum leakage specified, yields $R$ ) with the Flint approach (minimum but no maximum, yields $I$ ) and the Lim approach (minimum and maximum specified, yields ladj). A) In the Kreyling approach, the temperature at which a sample accrues $50 \%$ of possible damage (T50 for $R$ ) may not be meaningful; many samples may not reach this "Absolute 50\%" damage point. Instead, the temperature at which "Relative 50\%" damage is attained may be more meaningful. B) By comparison, values of $I$, in the Flint approach (orange line) are zeroed, although this may not drastically displace the curve relative to a Kreyling curve. C) In the Lim approach, data are forced to reach $100 \%$ damage, usually at the coldest temperature employed to freeze samples. Curve shapes may differ and critical temperatures (e.g., T50) corresponding to ladj (extracted from the Lim curve) may or may not differ from those associated with $R$ (Kreyling curve). D) Use of a liquid nitrogen control is expected to elevate all leakage values, making, for instance, a Kreyling curve behave more like a Lim curve and improving generalizability among approaches. 
A)

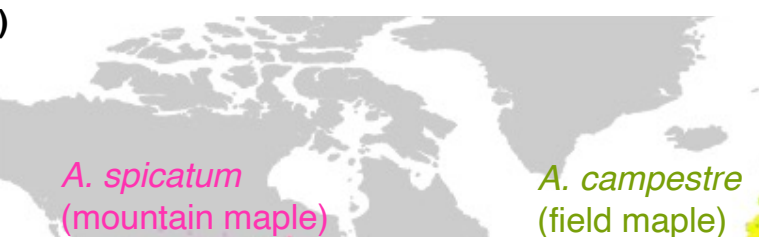

A. tegmentosum

(mountain maple) (field maple)

(Manchurian striped maple)

\section{A. saccharum (sugar maple)}

A. rubrum

(red maple)

\section{(box elder)}

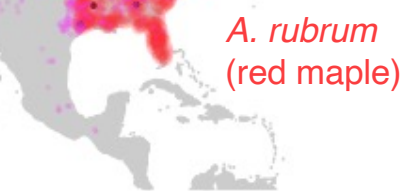

B)

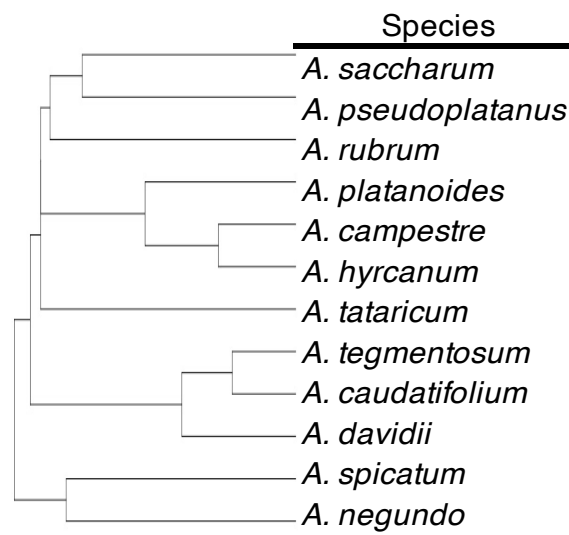

Section
Acer
Acer
Rubra
Platanoidea
Platanoidea
Acer
Ginnala
Macrantha
Macrantha
Macrantha
Spicata
Negundo

Region

A. davidii

(Father David's maple)

\section{A. hyrcanum}

(Balkan maple)
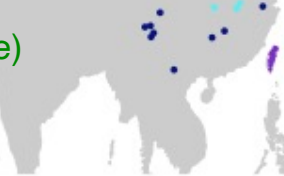

A. caudatifolium (kawakami maple)

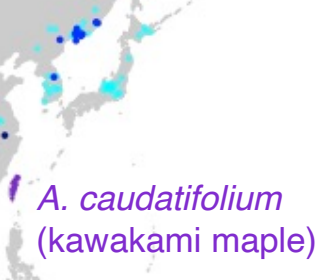

Figure 2. A) Native and naturalized distributions of the 12 maple study species, which are distributed across the North American (red/pink), European (green/yellow), and Asian (blue/purple) extent of the genus (Grossman, in review). Star indicates the location of the Arnold Arboretum. B) Phylogenetic relatedness and ecological descriptions for the study species. Phylogeny and section designations adapted from Li et al. (2019) and ArecesBerazain et al. (2020). 


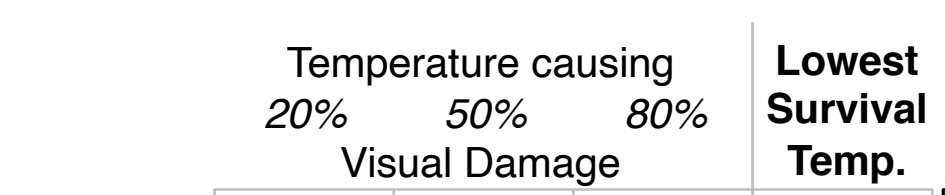

Kreyling $20 \%$

$\underset{50 \%}{\text { Kreyling }}$

Kreyling

\section{Flint}

$20 \%$

\section{Flint \\ $50 \%$}

\section{Flint $80 \%$}

\section{Lim $_{\text {logistic }}$ $20 \%$}

\section{Lim $_{\text {logistic }}$ $50 \%$}

\section{Lim $_{\text {logistic }}$ $80 \%$}

\section{Lim $_{\text {gomp. }}$ $20 \%$}

\section{Lim $_{\text {gomp. }}$ $50 \%$}

\section{Lim $_{\text {gomp. }}$ $80 \%$}

B)

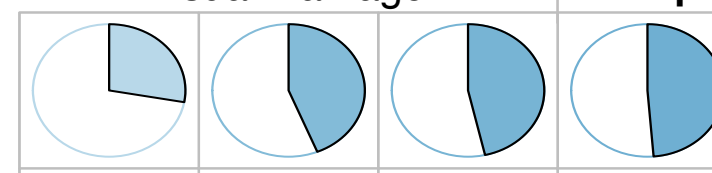

\section{D}
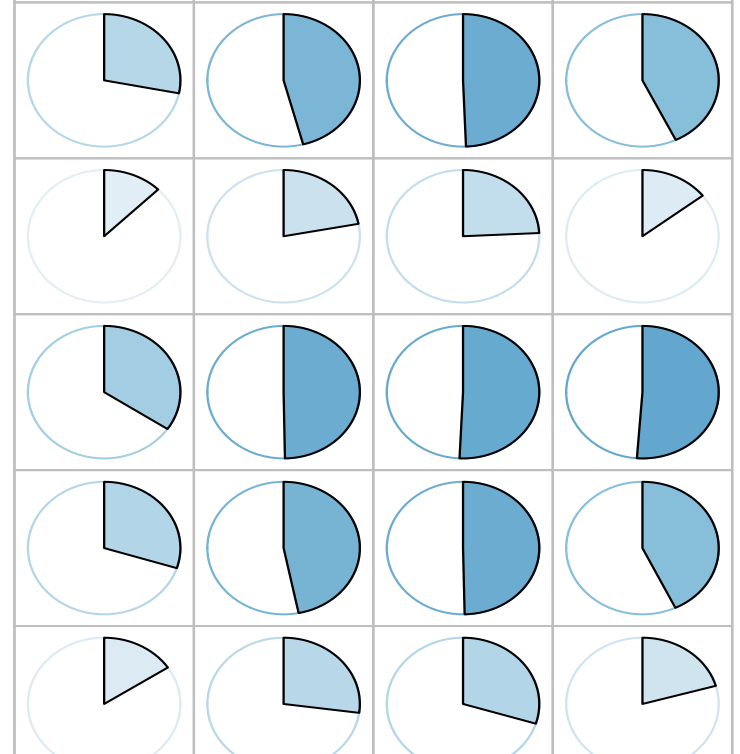

0.7
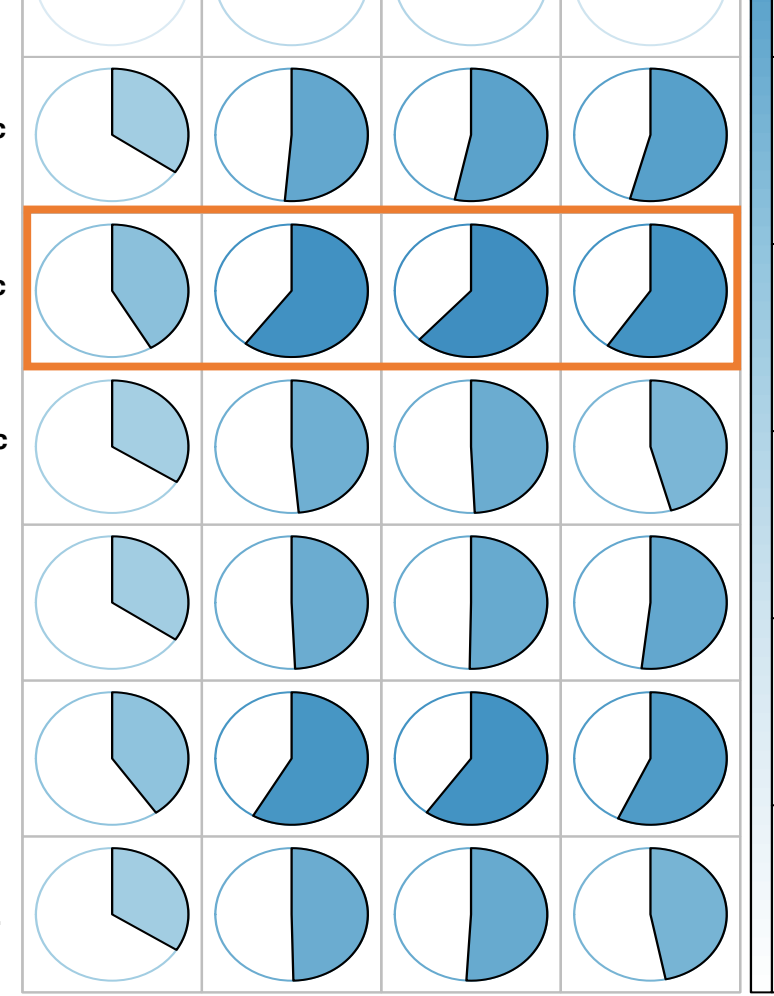

0.1
Figure 4. A) Validation of critical values from four approaches to modeling electrolyte leakage (as in Fig. 1) against visual estimates of freezing damage.

Critical values reflect either $20 \%, 50 \%$, or $80 \%$ electrolyte leakage (rows) or visual damage (columns). The rightmost column indicates lowest survival temperature (LST; Sakai et al. 1986), the lowest temperature at which stems experienced $<50 \%$ damage. Pie wedge size and color indicate correlation. B) $50 \%$ electrolyte leakage values using the Lim $_{\text {logistic }}$ approach (orange box) best predicted visual damage in the $40-60 \%$ damage range.

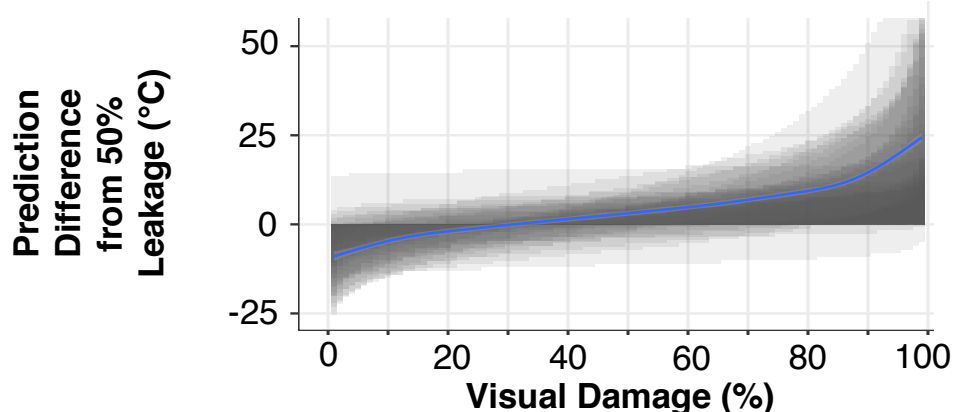




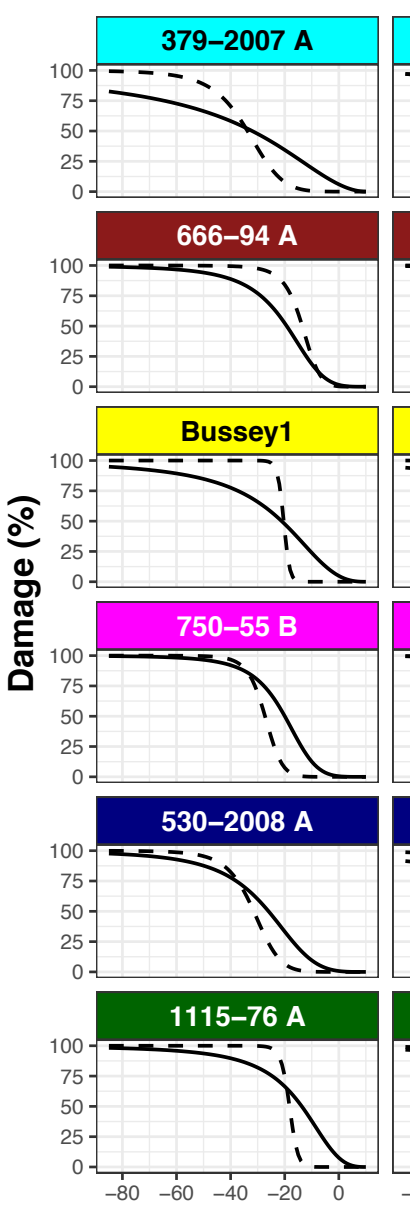

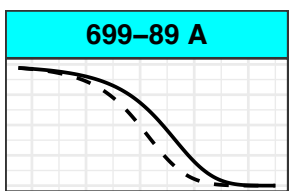
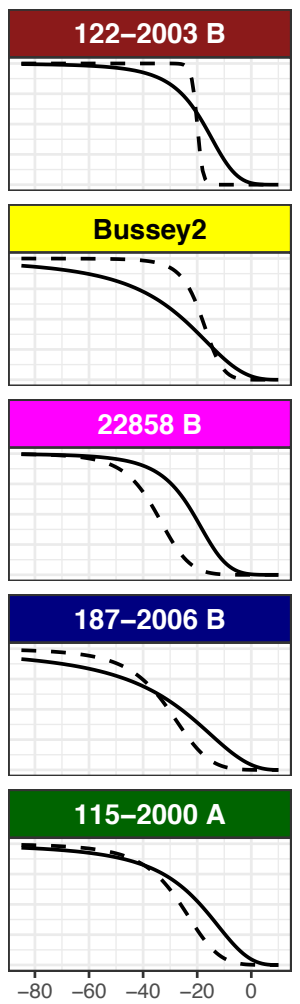
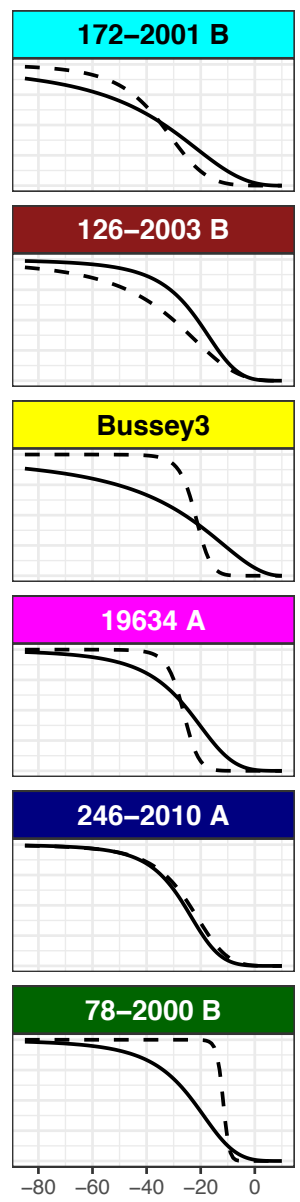

435-84 A
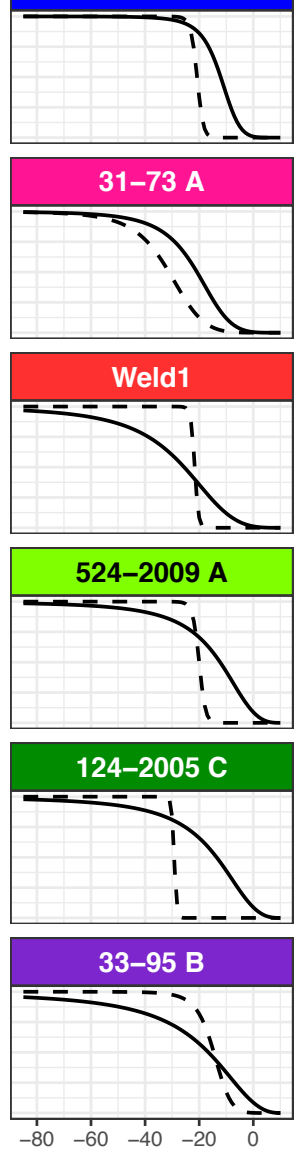

435-84 B
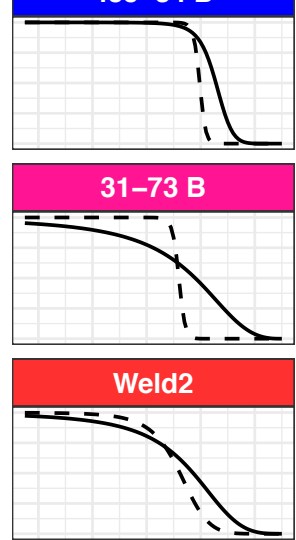

567-2008 B
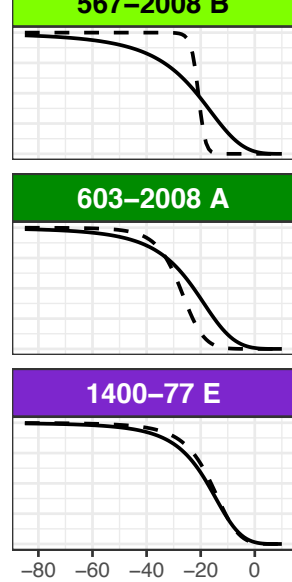

435-84 C
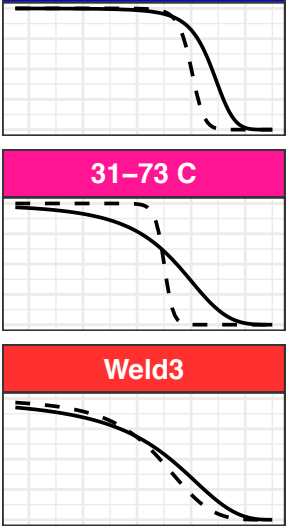

207-2005 A

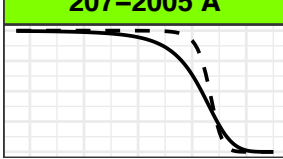

270-2010 A

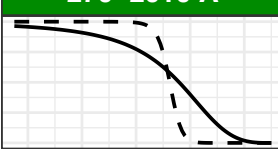

531-94 A

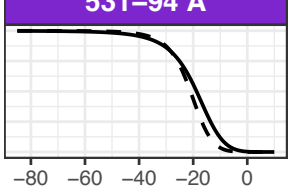

Temperature $\left({ }^{\circ} \mathrm{C}\right)$

Figure 5. Damage, as reflected by electrolyte leakage (solid lines) and visual estimates (dashed lines), induced by

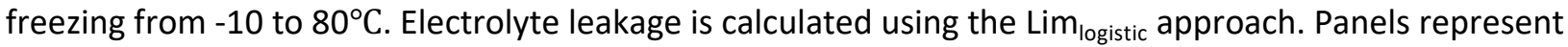
estimates of damage to particular genotypes. Color-coding indicates species as in Fig. 2; species are also presented in alphabetical order. 
Figure 6. Visual cambial damage corresponded to critical cold hardiness estimated from electrolyte leakage data.

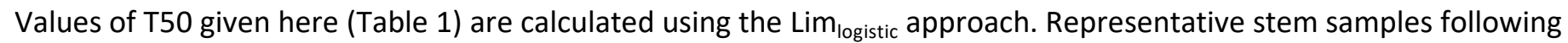
freezing are shown for A) Acer caudatifolium, B) A. davidii, C) A. hyrcanum, and D) A. negundo. Scale bar $=0.5 \mathrm{~cm}$.
A) Acer caudatifolium
$T_{50}=-13^{\circ} \mathrm{C}$
B) Acer davidii
$T_{50}=-19^{\circ} \mathrm{C}$

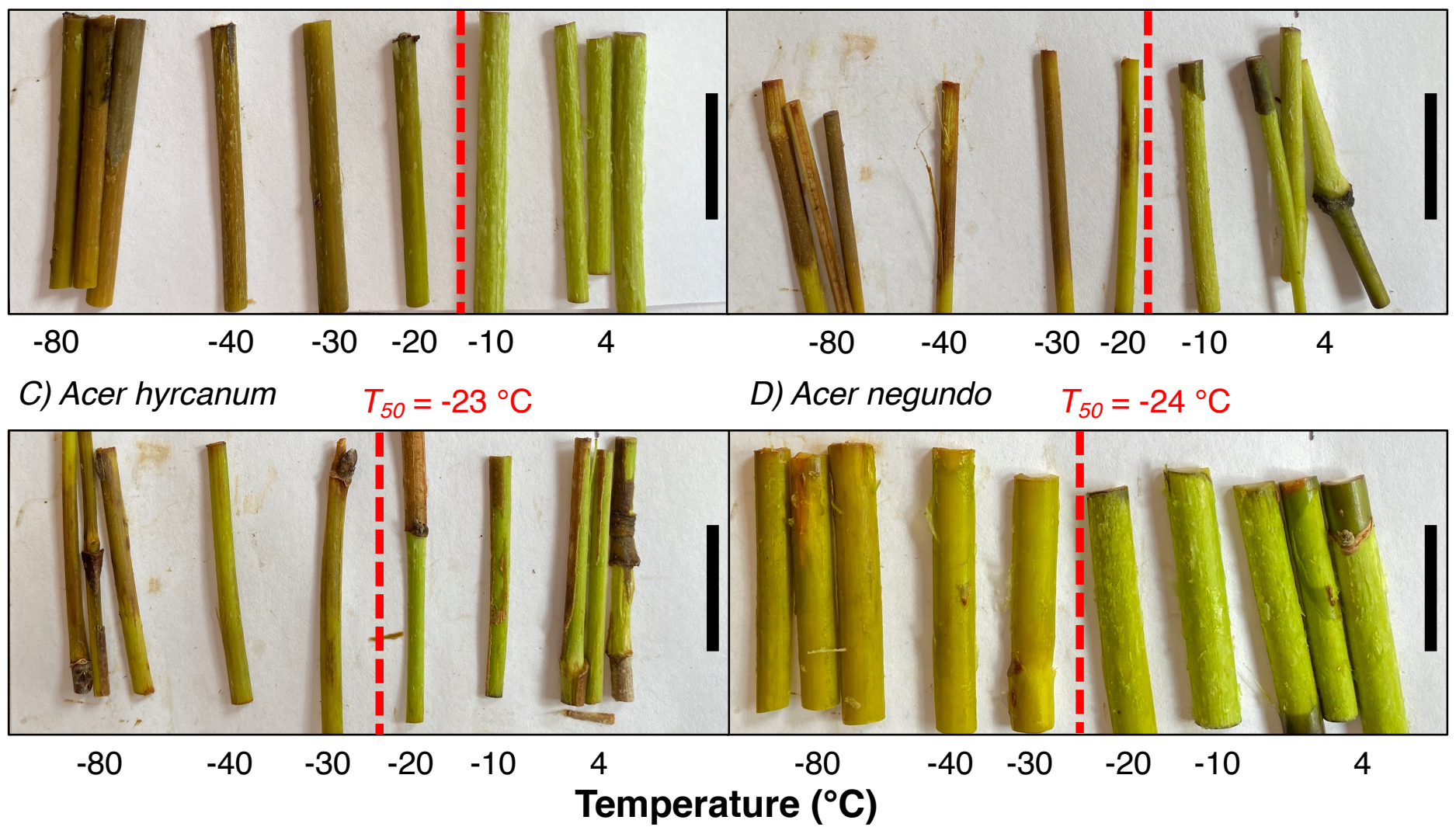




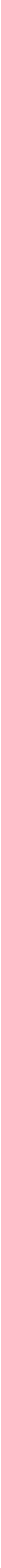

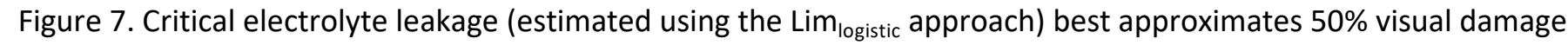
when leakage is between 50 and $80 \%$. Bias, though, is lowest from 20 to $50 \%$ leakage. Color-coding indicates species as in Fig. 2. Error reflects variation among genotypes of a given species. 


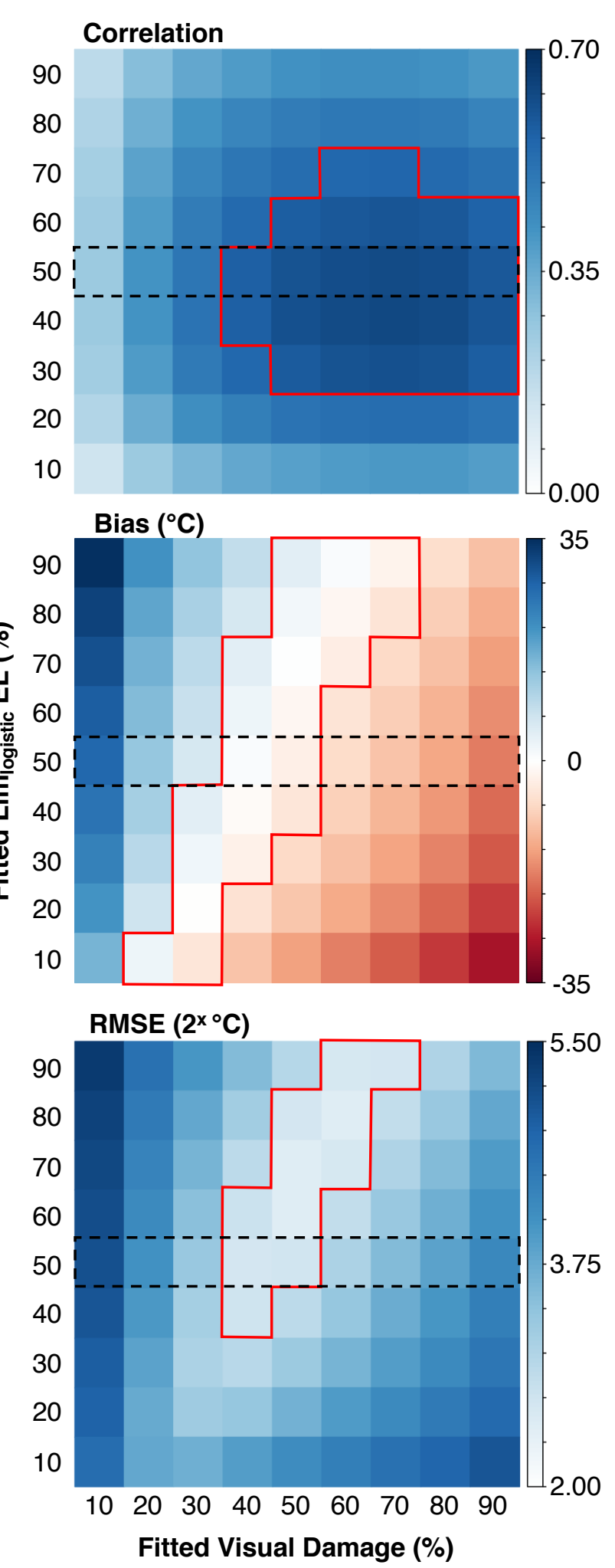

Figure 8. Fitness characteristics of the relationship between fitted values of electrolyte leakage and visual damage. Red contour delimits the area where: A) Correlation is greater than 0.55 ; B) Bias $<\operatorname{abs}\left(5^{\circ} \mathrm{C}\right)$; and $\mathrm{RMSE}<7^{\circ} \mathrm{C}$. Dashed rectangle delimits data used in Figure 7. 
A)
B)

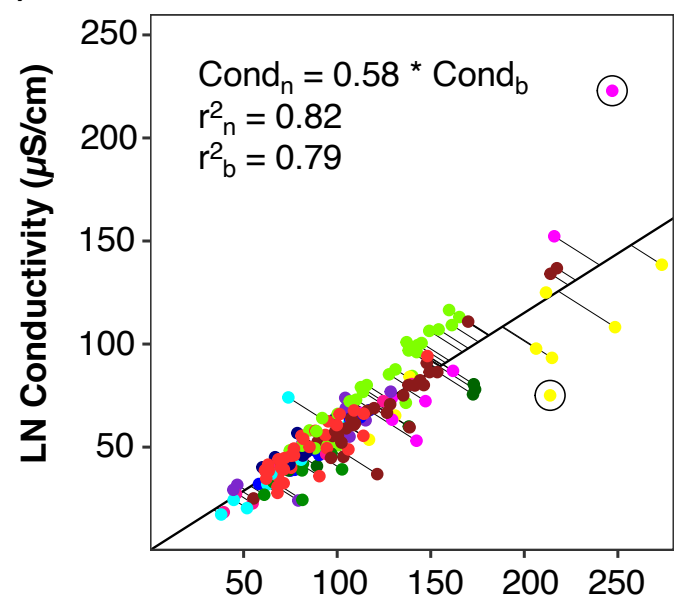

A)
B)

A)
B)

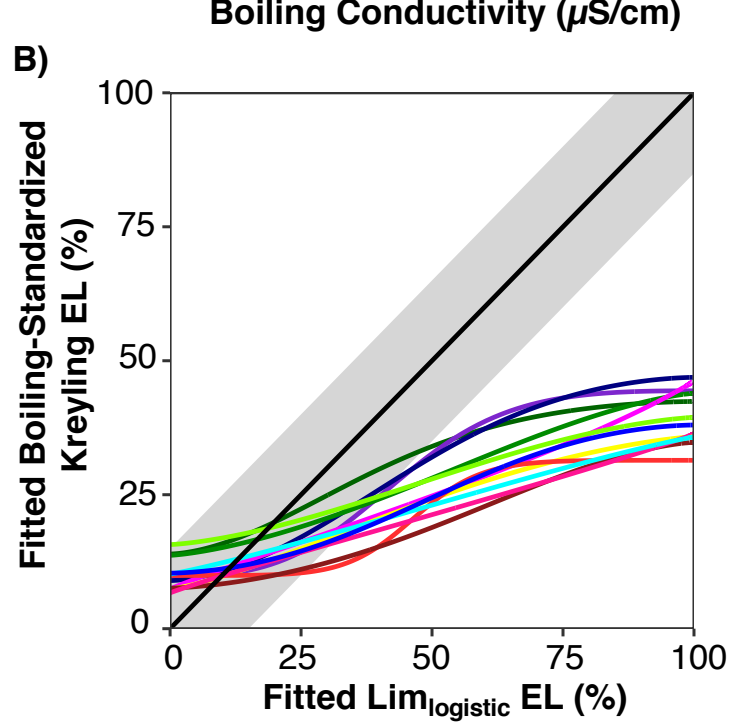

C)

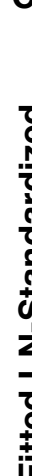

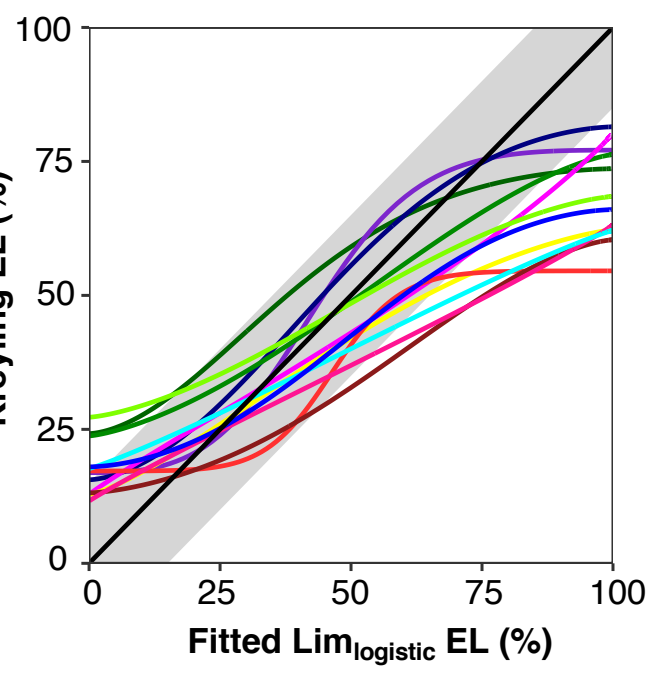

Figure 9. A) Sample conductivity following boiling predicts conductivity following immersion in liquid nitrogen across a range of values and for diverse species (color-coding indicates species as in Fig. 1.). Circled points are statistical outliers and lines indicate Deming regression error. $\mathrm{r}^{2}$ were calculated based on residuals in each direction. B) When a boiling standard is used, electrolyte leakage values derived using different curve-fitting procedures (e.g. Kreyling vs. $\operatorname{Lim}_{\text {logistic }}$ are not comparable above $25 \%$ leakage. C) However, use of a liquid nitrogen standard makes outputs of these two routines more comparable. Grey bar indicates a range of values within $15 \%$ of the $1: 1$ line. 
Boiling
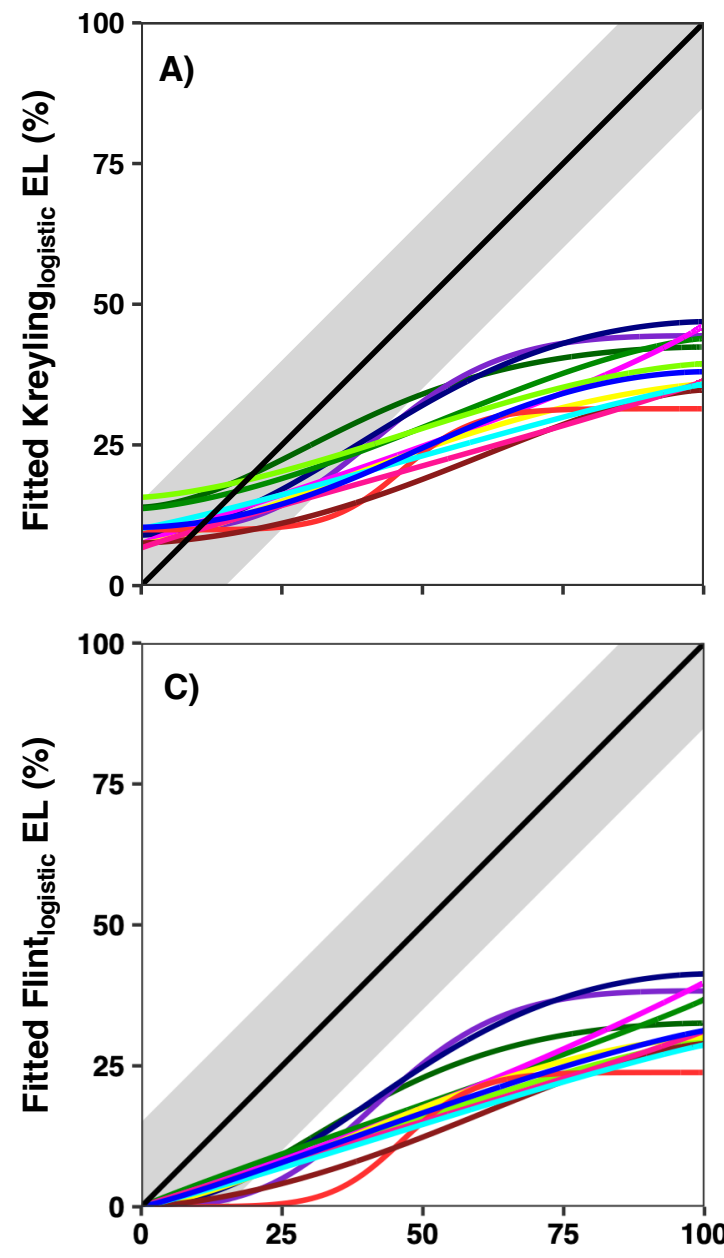

\section{Liquid $\mathbf{N}$}
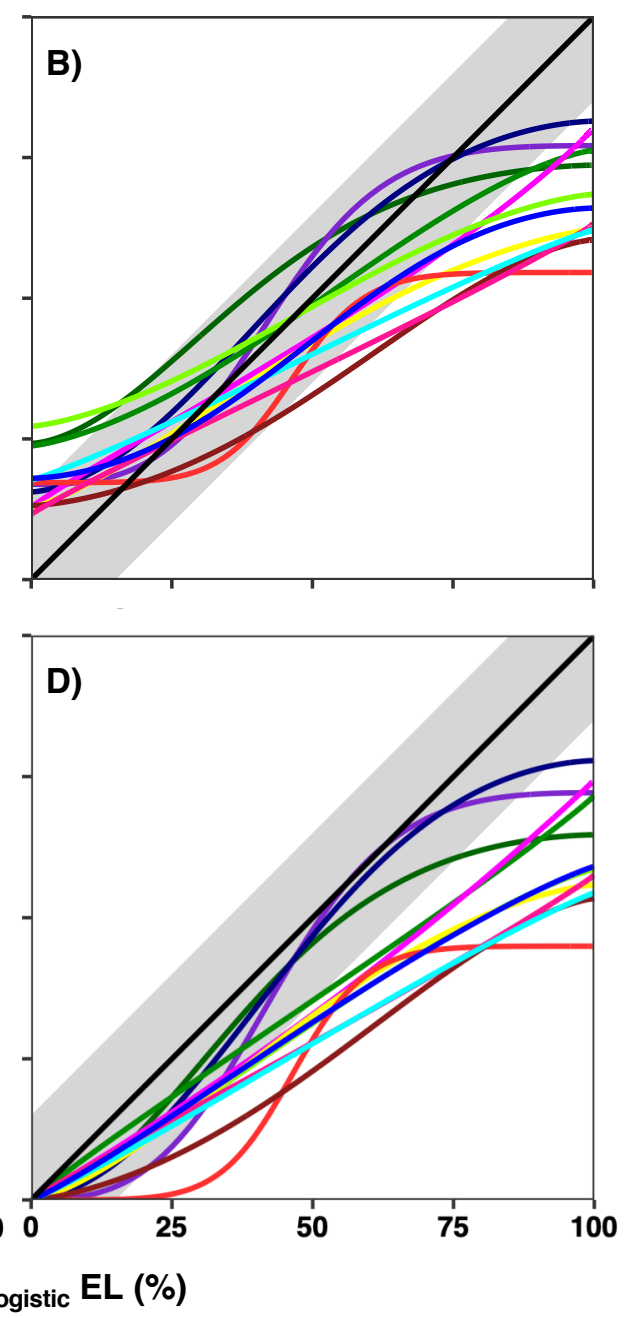

- CAMP

- CAUD

- DAVI

- HYRC

- NEGU

- PLAT PSEP

- RUBR SACC

- SPIC

- TATA

- TEGM

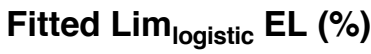

Figure 10. When a boiling standard is used, electrolyte leakage values derived using different curve-fitting procedures (e.g. Kreyling vs. Limlogistic vs. Flint approaches) are not comparable above $\sim 25 \%$ leakage (A vs. C). However, use of a liquid nitrogen standard makes outputs of these two routines more comparable (B vs. D). Grey bar indicates a range of values within $15 \%$ of the $1: 1$ line. 

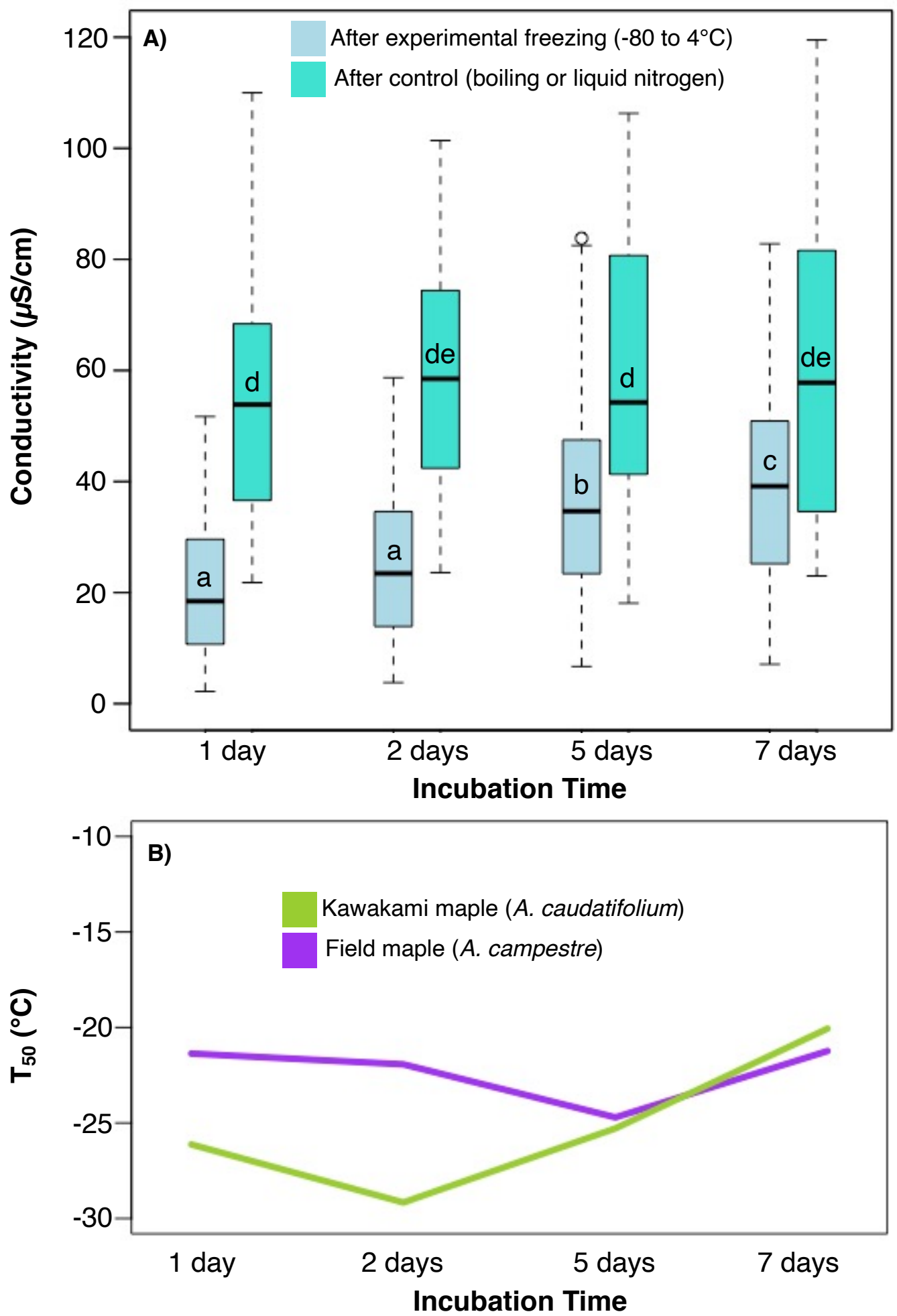

Figure 11. A) Electrolyte leakage increased gradually over seven days following experimental freezing (light blue), but not exposure to a boiling or liquid nitrogen control (turquoise), with no evidence of a significant difference when conductivity was measured over the first 48 hours after freezing. Lowercase letters indicate significant differences in conductivity measured at different time points at the 0.05 level based on models reported in Additional file 3 panels A (light blue, a-c) and B (turquoise, d-e). B) As a result of this pattern, estimates of critical values for cold hardiness $\left(T_{50}\right)$ are consistent and reflect species differences when samples were incubated for one or two days, but not when they were incubated for 5 or 7 days (Additional file 4). 
Figure 12. Stem segments incubated for longer than five days following control treatment (boiling or liquid nitrogen immersion) tended to deteriorate, showing evidence of microbial growth.

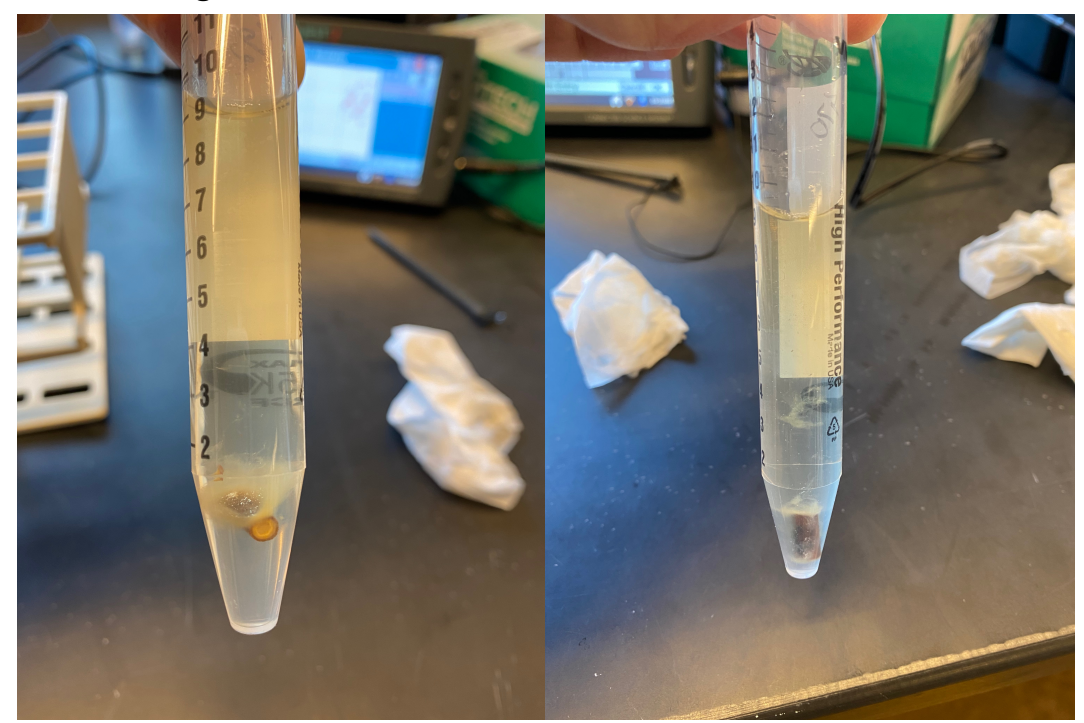


Figures

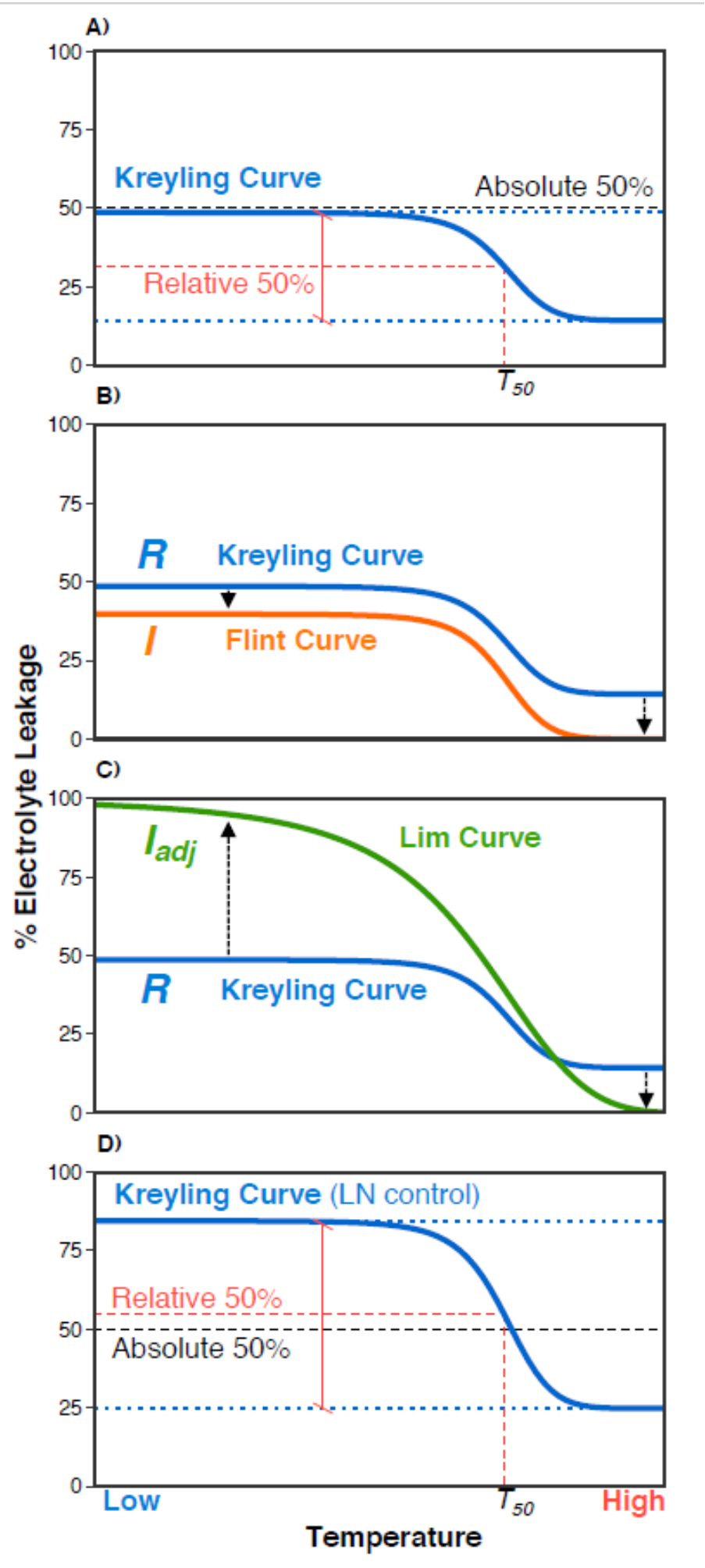

\section{Figure 1}

Schematic comparing different approaches to measuring electrolyte leakage. As described in the main text (Fig. 2), we contrast the Kreyling approach (no minimum or maximum leakage specified, yields R) with the Flint approach (minimum but no maximum, yields I) and the Lim approach (minimum and 
maximum specified, yields ladj). A) In the Kreyling approach, the temperature at which a sample accrues $50 \%$ of possible damage ( $\mathrm{T} 50$ for R) may not be meaningful; many samples may not reach this "Absolute $50 \%$ " damage point. Instead, the temperature at which "Relative $50 \%$ " damage is attained may be more meaningful. B) By comparison, values of I, in the Flint approach (orange line) are zeroed, although this may not drastically displace the curve relative to a Kreyling curve. C) In the Lim approach, data are forced to reach $100 \%$ damage, usually at the coldest temperature employed to freeze samples. Curve shapes may differ and critical temperatures (e.g., T50) corresponding to ladj (extracted from the Lim curve) may or may not differ from those associated with $\mathrm{R}$ (Kreyling curve). D) Use of a liquid nitrogen control is expected to elevate all leakage values, making, for instance, a Kreyling curve behave more like a Lim curve and improving generalizability among approaches.

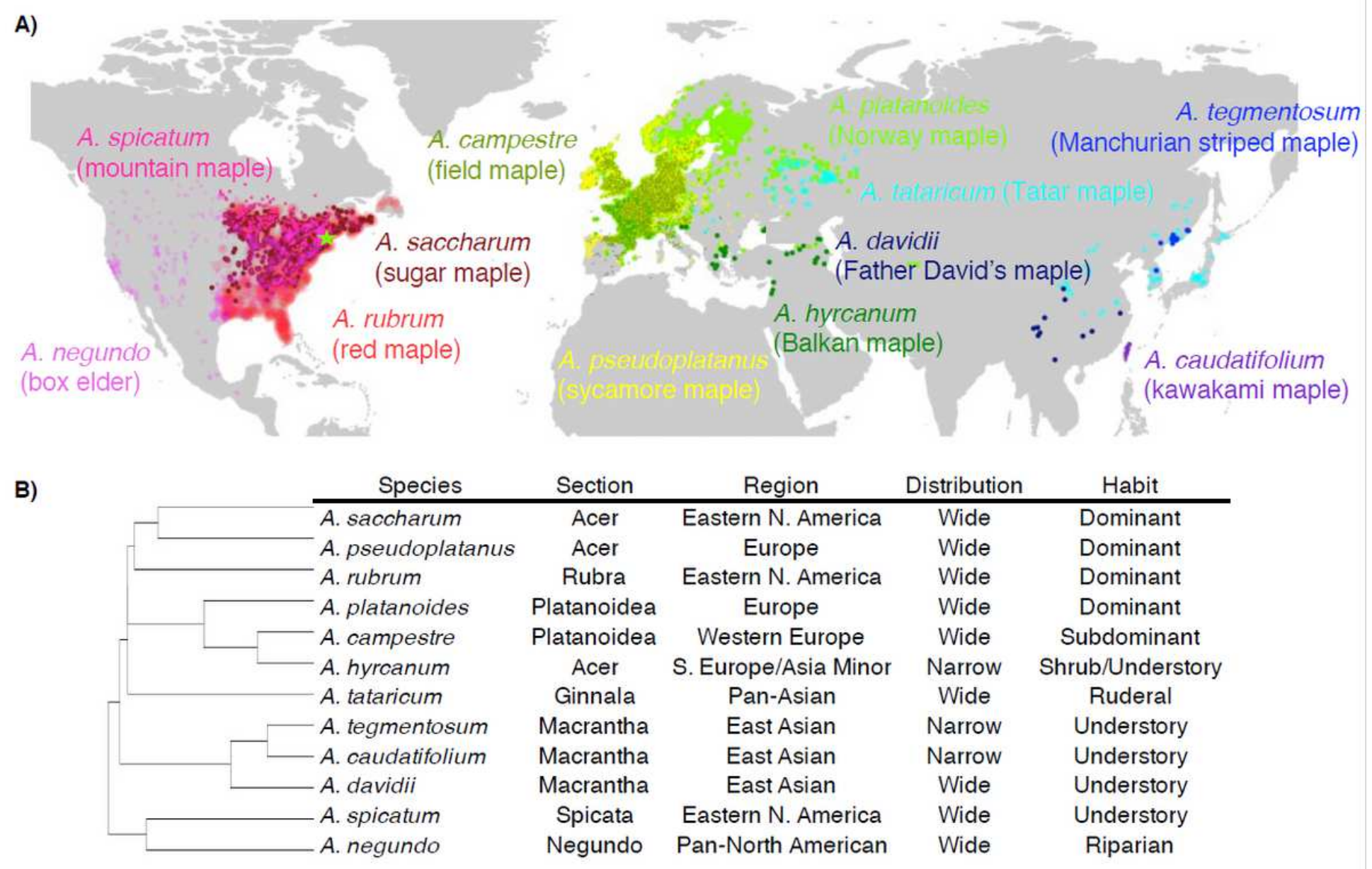

\section{Figure 2}

Schematic comparing different approaches to measuring electrolyte leakage. As described in the main text (Fig. 2), we contrast the Kreyling approach (no minimum or maximum leakage specified, yields R) with the Flint approach (minimum but no maximum, yields $\mathrm{I}$ ) and the Lim approach (minimum and maximum specified, yields ladj). A) In the Kreyling approach, the temperature at which a sample accrues $50 \%$ of possible damage ( $\mathrm{T} 50$ for R) may not be meaningful; many samples may not reach this "Absolute $50 \%$ " damage point. Instead, the temperature at which "Relative $50 \%$ " damage is attained may be more meaningful. B) By comparison, values of I, in the Flint approach (orange line) are zeroed, although this 
may not drastically displace the curve relative to a Kreyling curve. C) In the Lim approach, data are forced to reach $100 \%$ damage, usually at the coldest temperature employed to freeze samples. Curve shapes may differ and critical temperatures (e.g., T50) corresponding to ladj (extracted from the Lim curve) may or may not differ from those associated with $\mathrm{R}$ (Kreyling curve). D) Use of a liquid nitrogen control is expected to elevate all leakage values, making, for instance, a Kreyling curve behave more like a Lim curve and improving generalizability among approaches. Note: The designations employed and the presentation of the material on this map do not imply the expression of any opinion whatsoever on the part of Research Square concerning the legal status of any country, territory, city or area or of its authorities, or concerning the delimitation of its frontiers or boundaries. This map has been provided by the authors. 
A. campestre
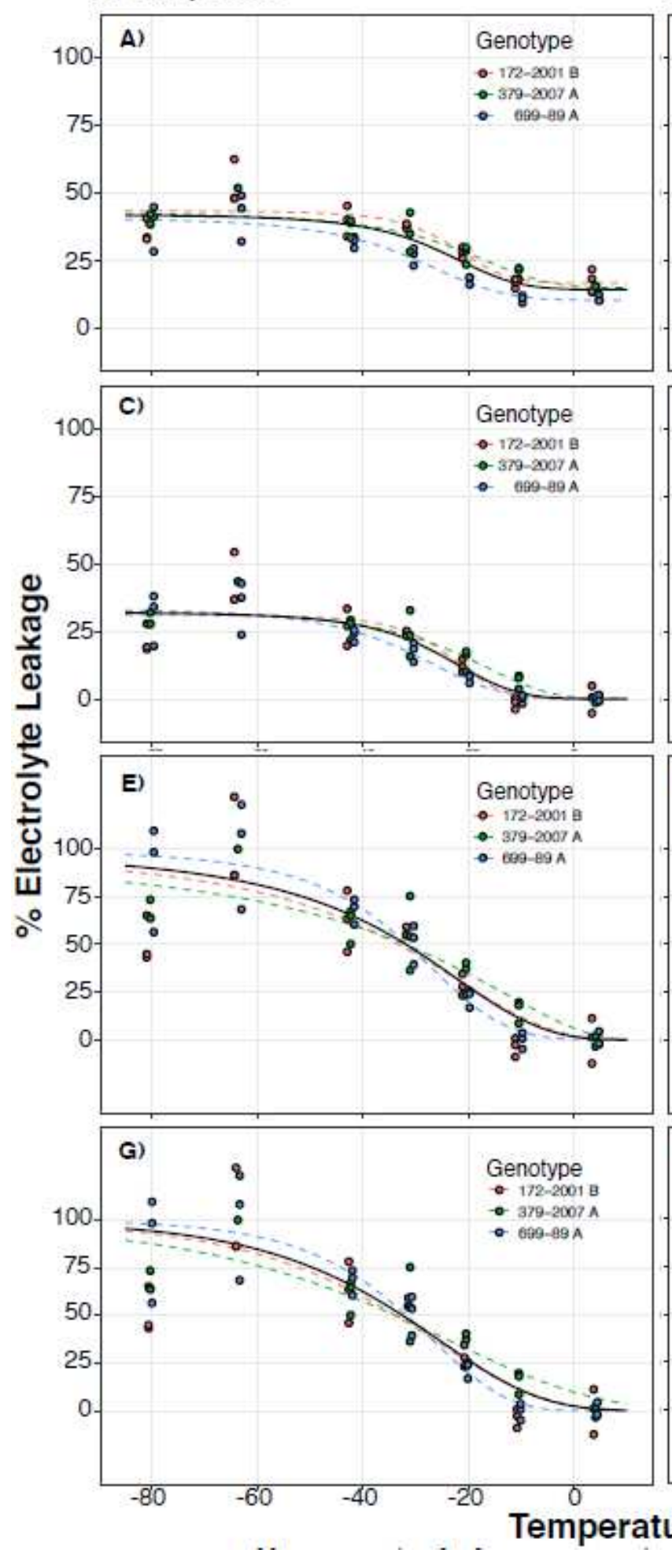

A. caudatifolium

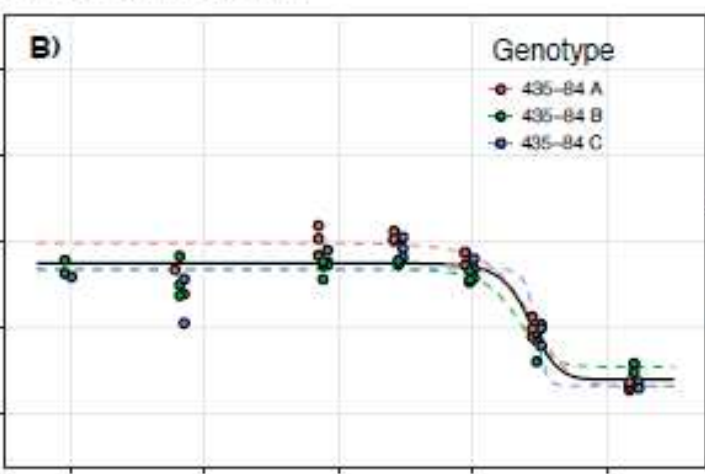

Kreyling no zero no $\max$. logistic

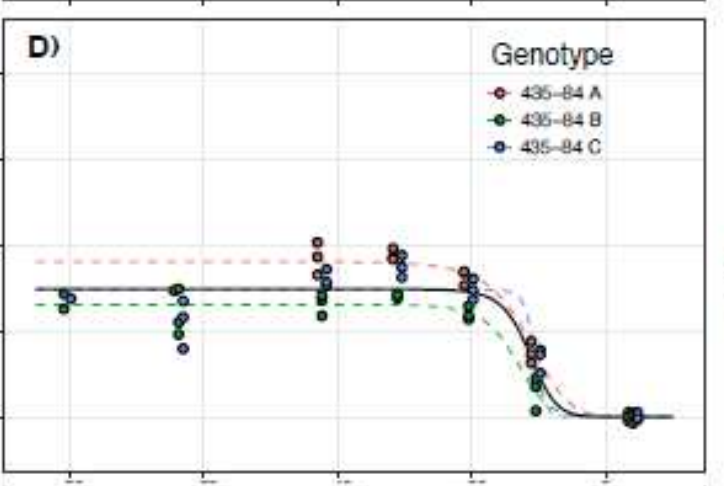

Flint

zeroed no max. logistic
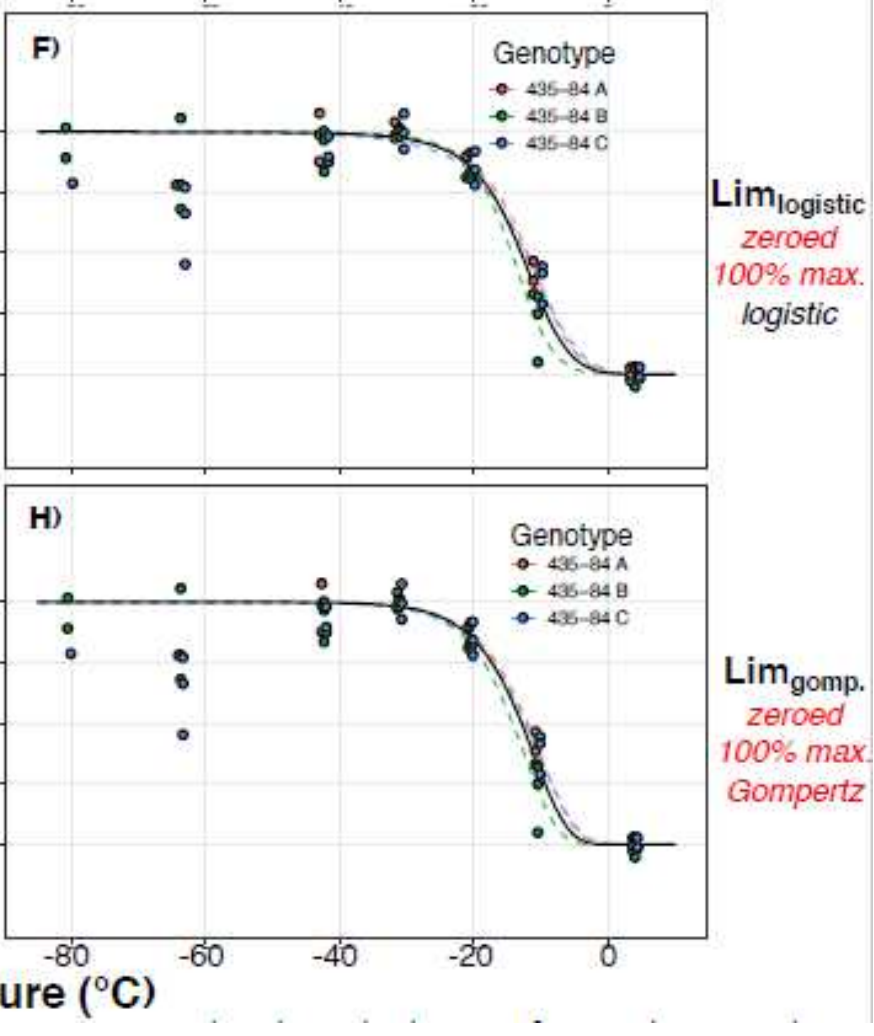

\section{Figure 3}

Comparison of four approaches for fitting curves to data representing the relationship between freezing damage and temperature in $(A, C, E, G) A$. caudatifolium and $(B, D, F, H) A$. campestre stem segments (plots for other species provided in Appendix 1). Curves fit to data on a per-genotype (red, blue, and green) and per-species (black curve) basis are fit in each case. Panels show curves fit following the approach of A, B) Kreyling and colleagues' (2015), C, D) Flint et al. (1987), and Lim et al. (1998). Approaches vary, as 
indicated, in their use of room-temperature (zeroing; $\mathrm{C}-\mathrm{H}$ ) and deep freezing (maximum damage; $\mathrm{E}-\mathrm{H}$ ) controls and reliance on general logistic (A-F) vs. Gompertz (G-H) curves.

A)

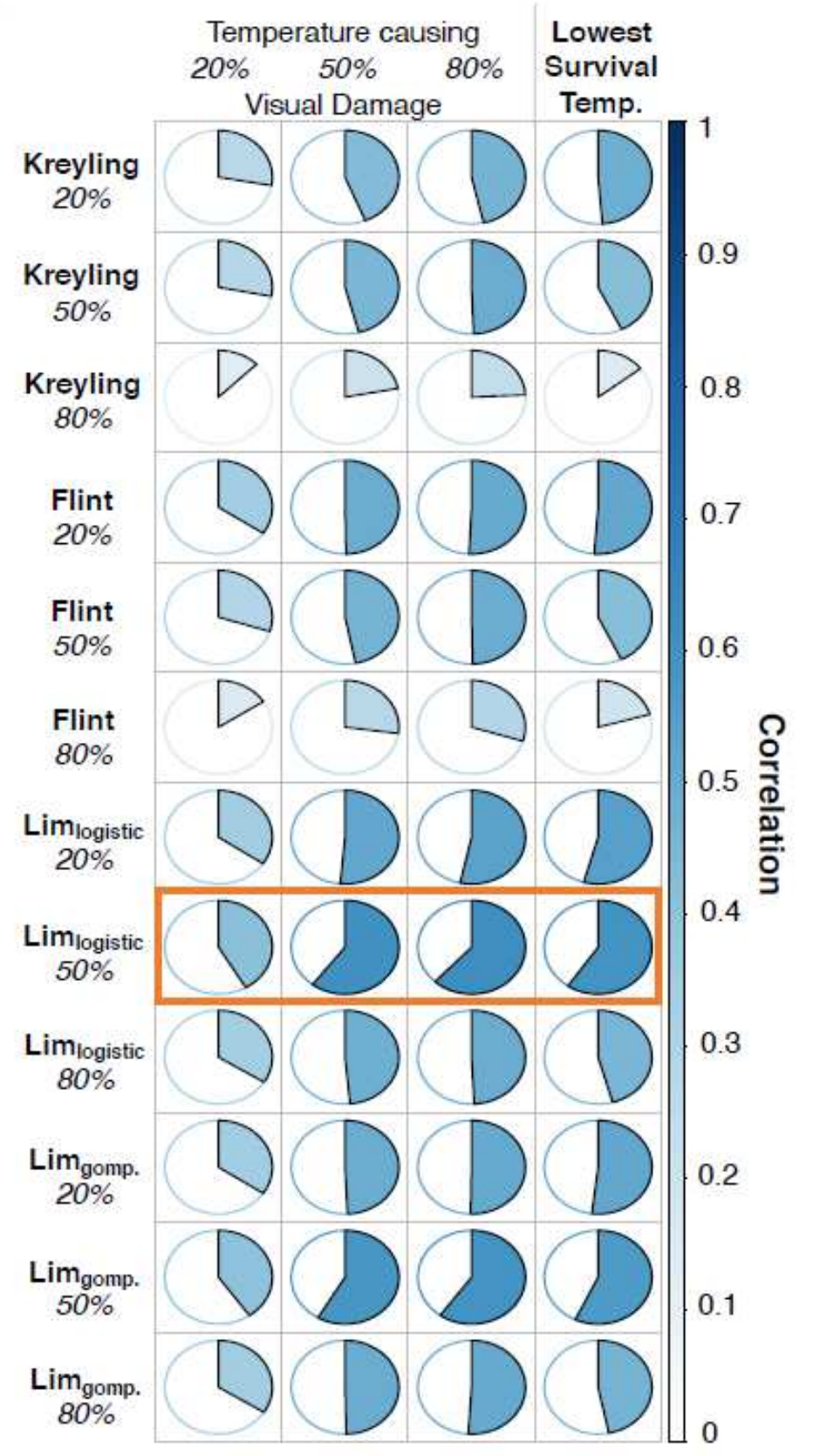

B)

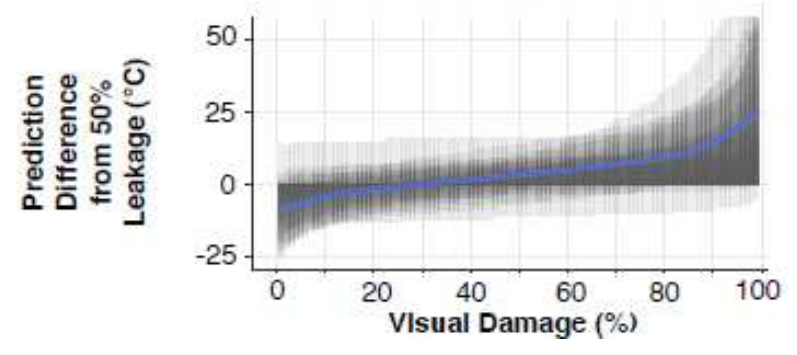

Figure 4

A) Validation of critical values from four approaches to modeling electrolyte leakage (as in Fig. 1) against visual estimates of freezing damage. Critical values reflect either $20 \%, 50 \%$, or $80 \%$ electrolyte leakage (rows) or visual damage (columns). The rightmost column indicates lowest survival temperature (LST), 
the lowest temperature at which stems experienced $<50 \%$ damage. Pie wedge size and color indicate correlation. B) 50\% electrolyte leakage values using the Limlogistic approach (orange box) best predicted visual damage in the $40-60 \%$ damage range.

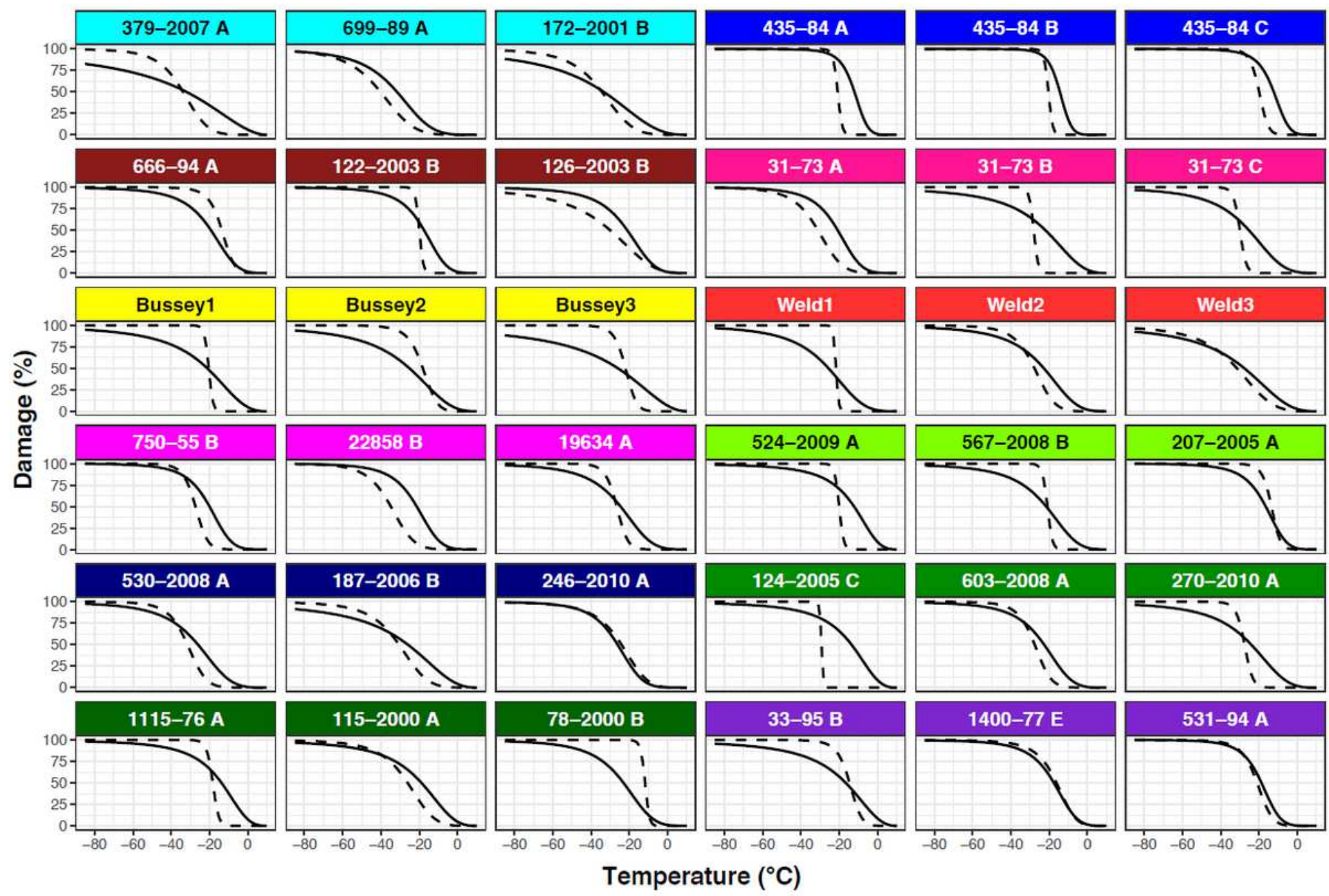

Figure 5

Damage, as reflected by electrolyte leakage (solid lines) and visual estimates (dashed lines), induced by freezing from -10 to $80 \rrbracket$. Electrolyte leakage is calculated using the Limlogistic approach. Panels represent estimates of damage to particular genotypes. Color-coding indicates species as in Fig. 2; species are also presented in alphabetical order. 


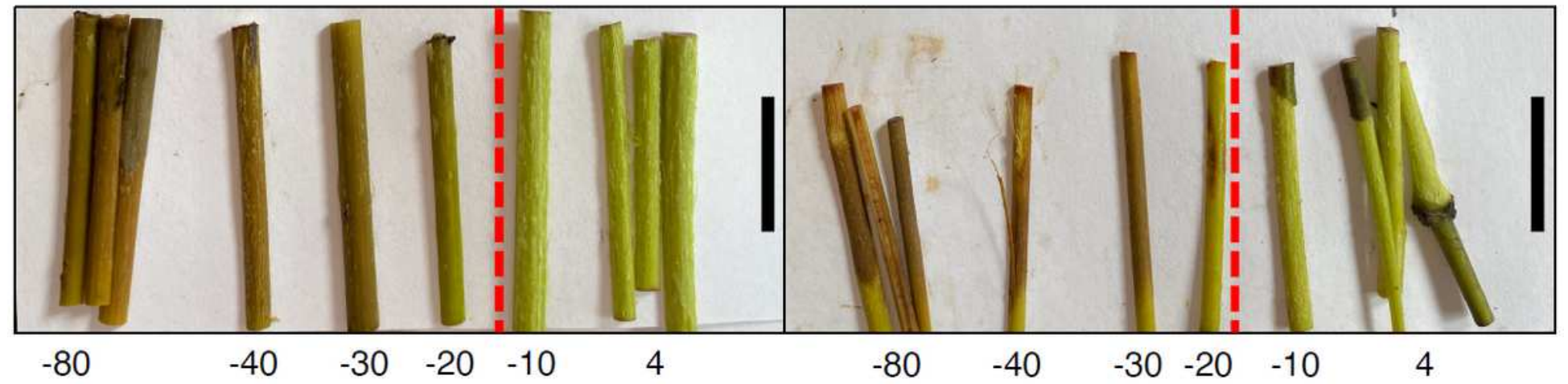

C) Acer hyrcanum

$$
T_{50}=-23^{\circ} \mathrm{C}
$$

D) Acer negundo

$T_{50}=-24^{\circ} \mathrm{C}$

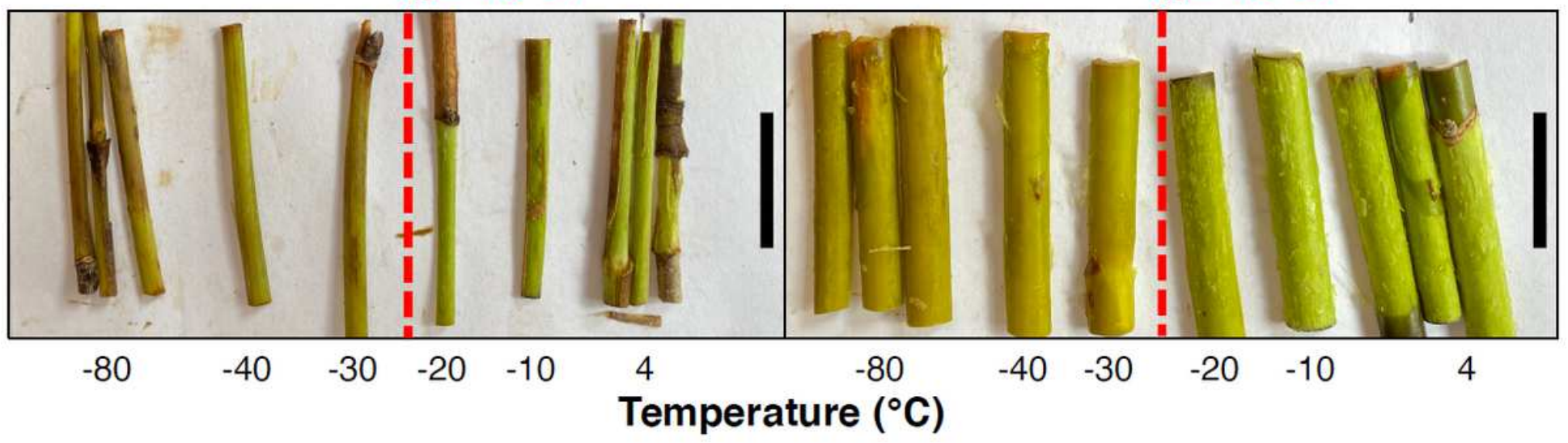

Figure 6

Visual cambial damage corresponded to critical cold hardiness estimated from electrolyte leakage data. Values of T50 given here (Table 1) are calculated using the Limlogistic approach. Representative stem samples following freezing are shown for A) Acer caudatifolium, B) A. davidii, C) A. hyrcanum, and D) A. negundo. Scale bar $=0.5 \mathrm{~cm}$. 


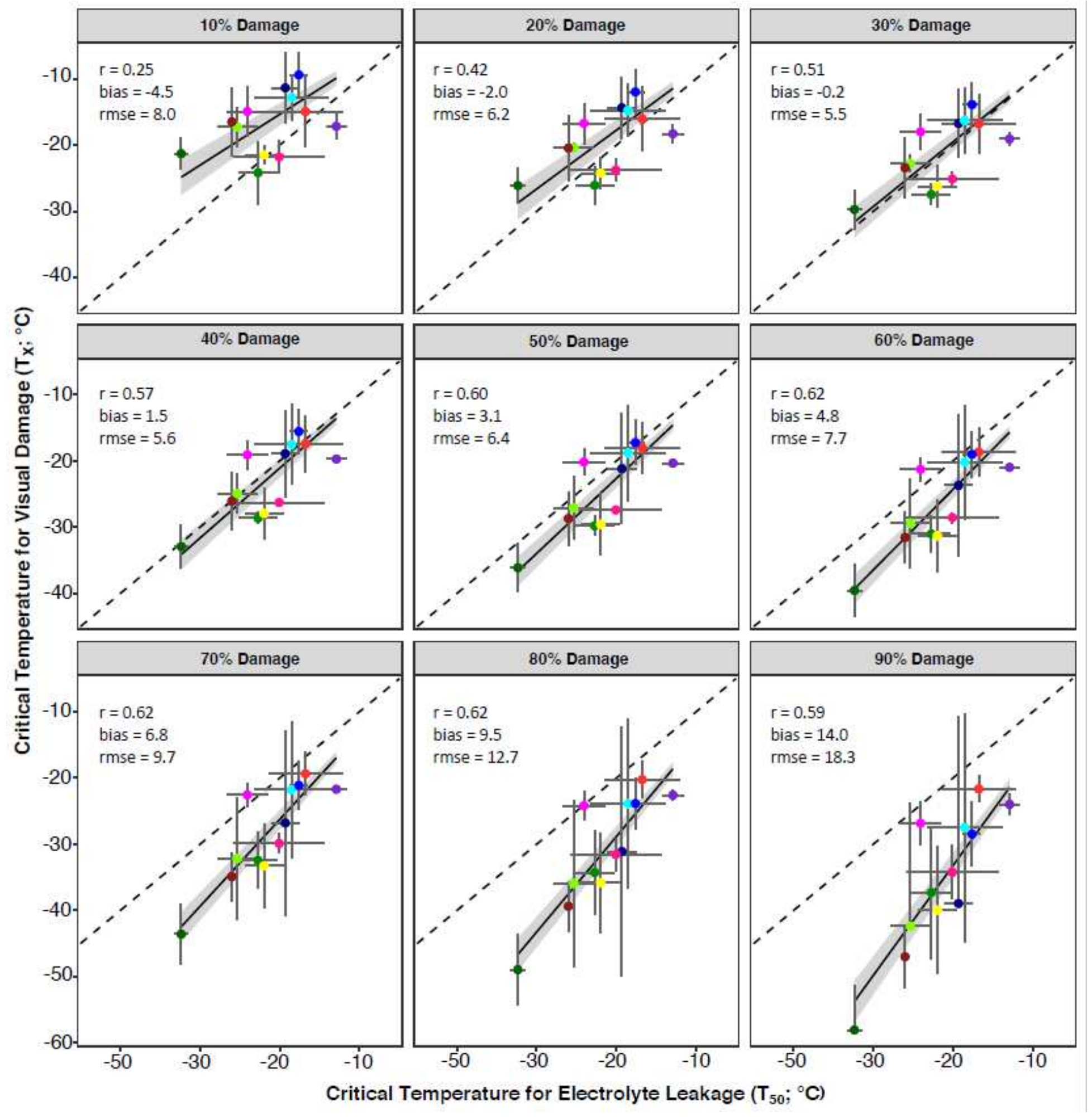

Figure 7

Critical electrolyte leakage (estimated using the Limlogistic approach) best approximates 50\% visual damage when leakage is between 50 and $80 \%$. Bias, though, is lowest from 20 to $50 \%$ leakage. Colorcoding indicates species as in Fig. 2. Error reflects variation among genotypes of a given species. 


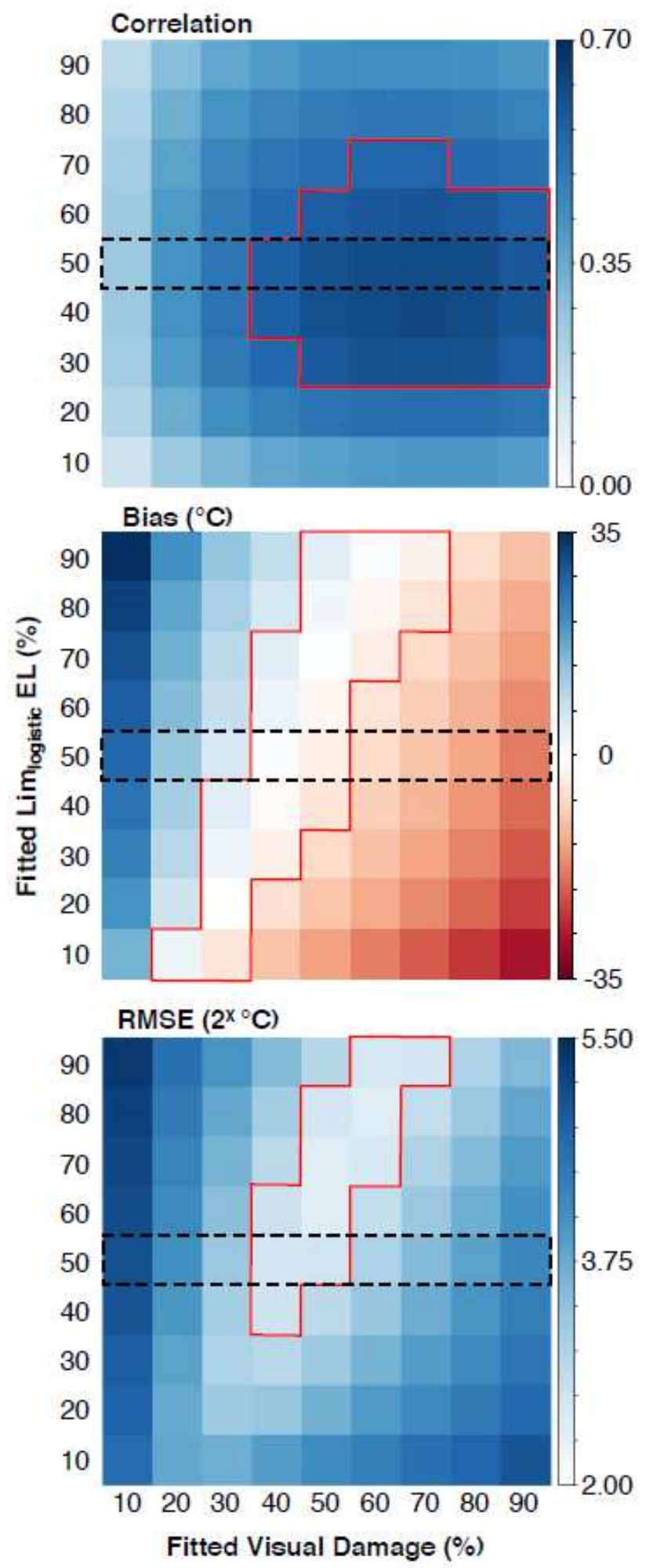

Figure 8

Fitness characteristics of the relationship between fitted values of electrolyte leakage and visual damage.

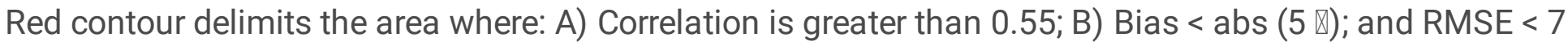
\. Dashed rectangle delimits data used in Figure 7. 
A)

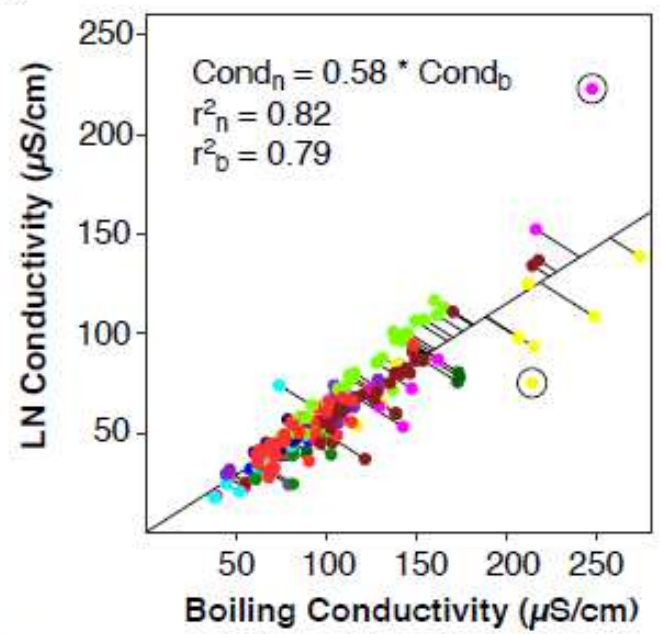

B)
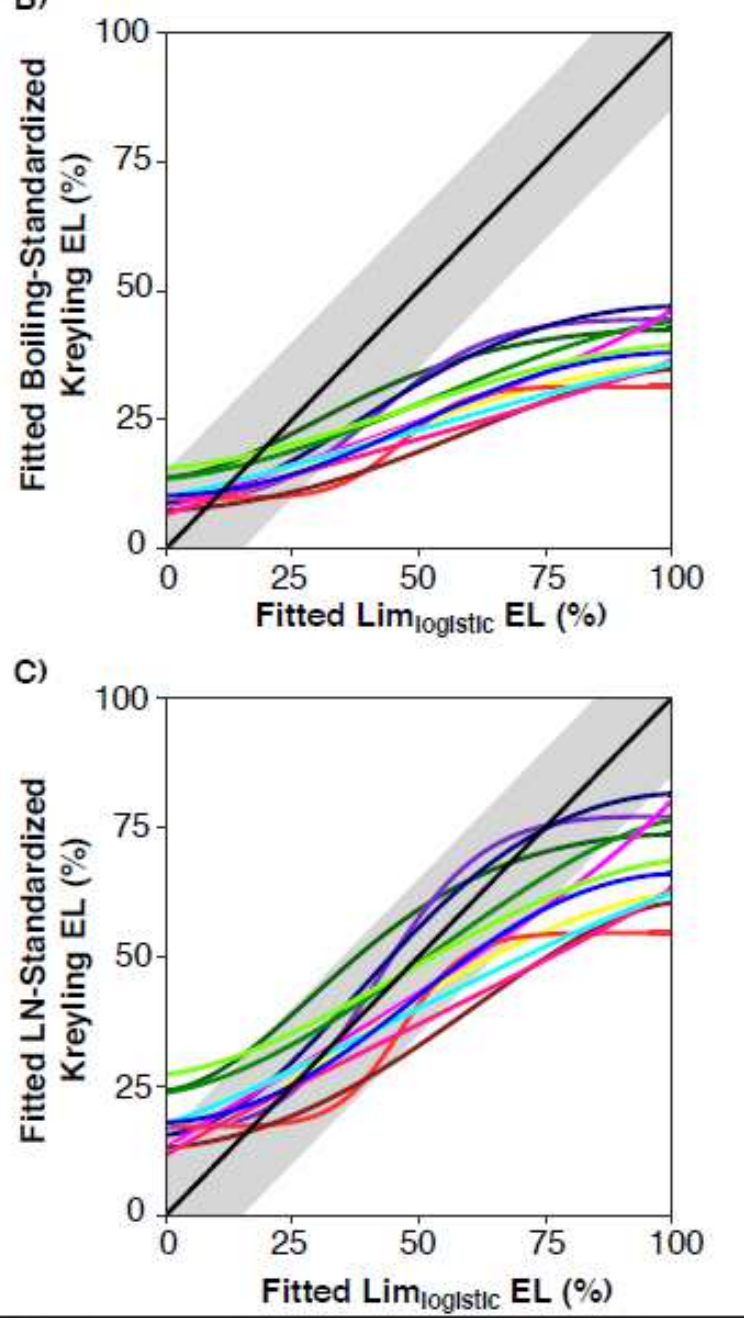

Figure 9

A) Sample conductivity following boiling predicts conductivity following immersion in liquid nitrogen across a range of values and for diverse species (color-coding indicates species as in Fig. 2.). Circled points are statistical outliers and lines indicate Deming regression error. r2 were calculated based on residuals in each direction. B) When a boiling standard is used, electrolyte leakage values derived using different curve-fitting procedures (e.g. Kreyling vs. Limlogistic) are not comparable above $\sim 25 \%$ leakage. 
C) However, use of a liquid nitrogen standard makes outputs of these two routines more comparable. Grey bar indicates a range of values within $15 \%$ of the $1: 1$ line.

Boiling
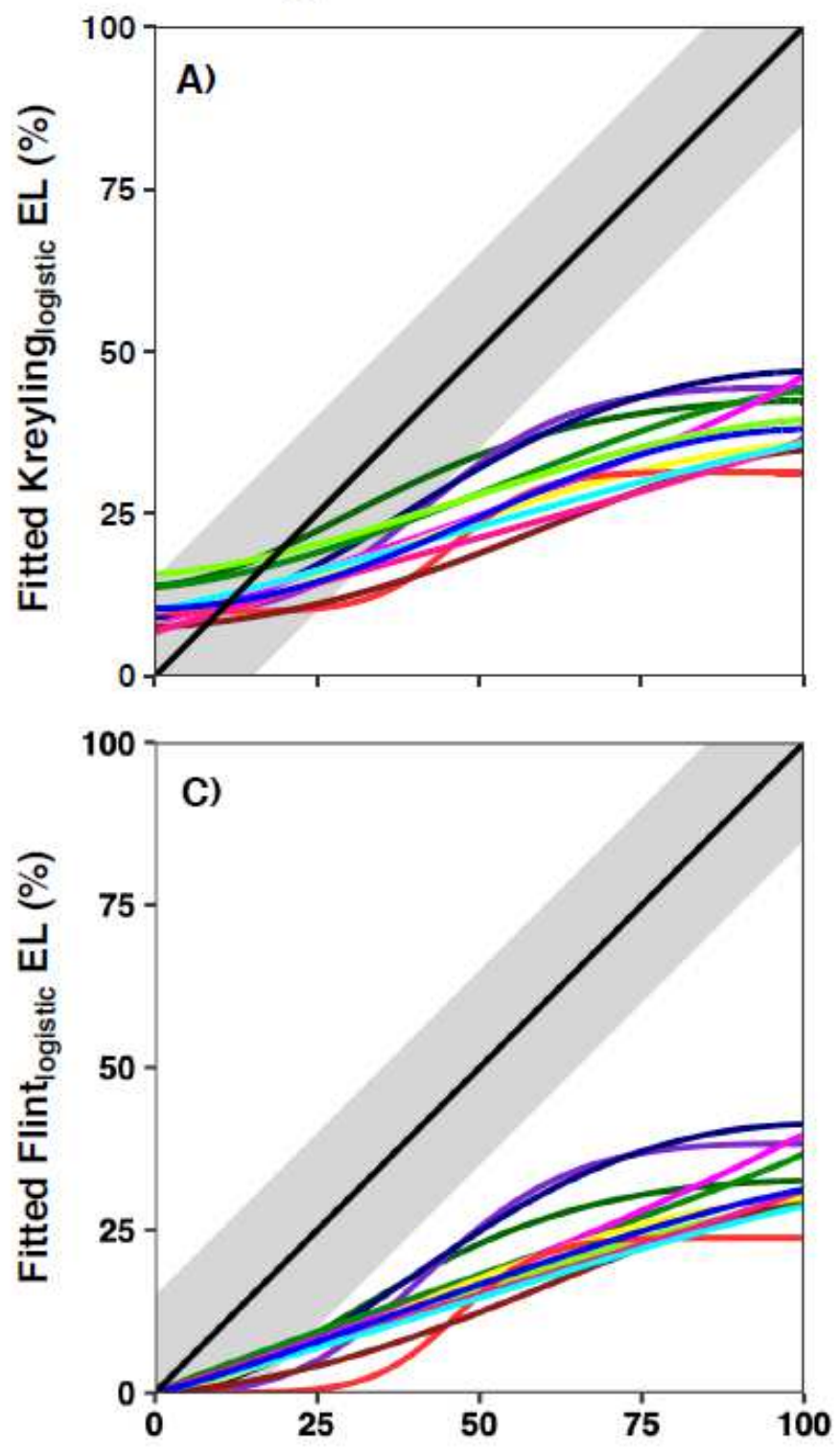

\section{Liquid $\mathrm{N}$}

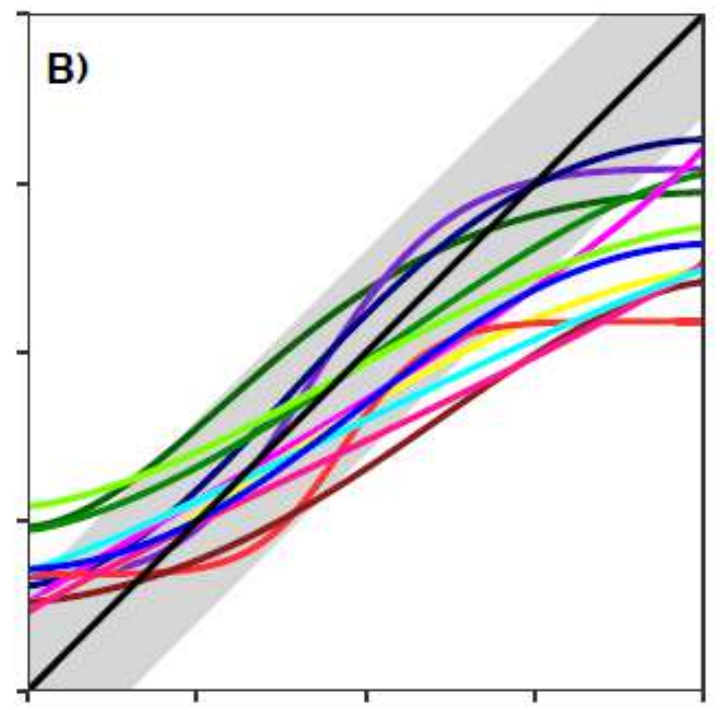

- CAMP

- CAUD

- DAVI

- HYRC

- NEGU

- PLAT

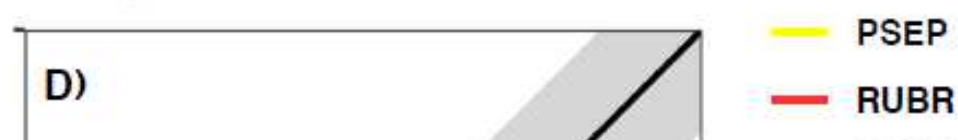

- RUBR

- SACC

- SPIC

- TATA

— TEGM

Fitted Lim loglstlc $_{\text {EL }(\%)}$

Figure 10

When a boiling standard is used, electrolyte leakage values derived using different curve-fitting procedures (e.g. Kreyling vs. Limlogistic vs. Flint approaches) are not comparable above $25 \%$ leakage (A vs. B). However, use of a liquid nitrogen standard makes outputs of these two routines more comparable (C vs. D). Grey bar indicates a range of values within $15 \%$ of the $1: 1$ line. 

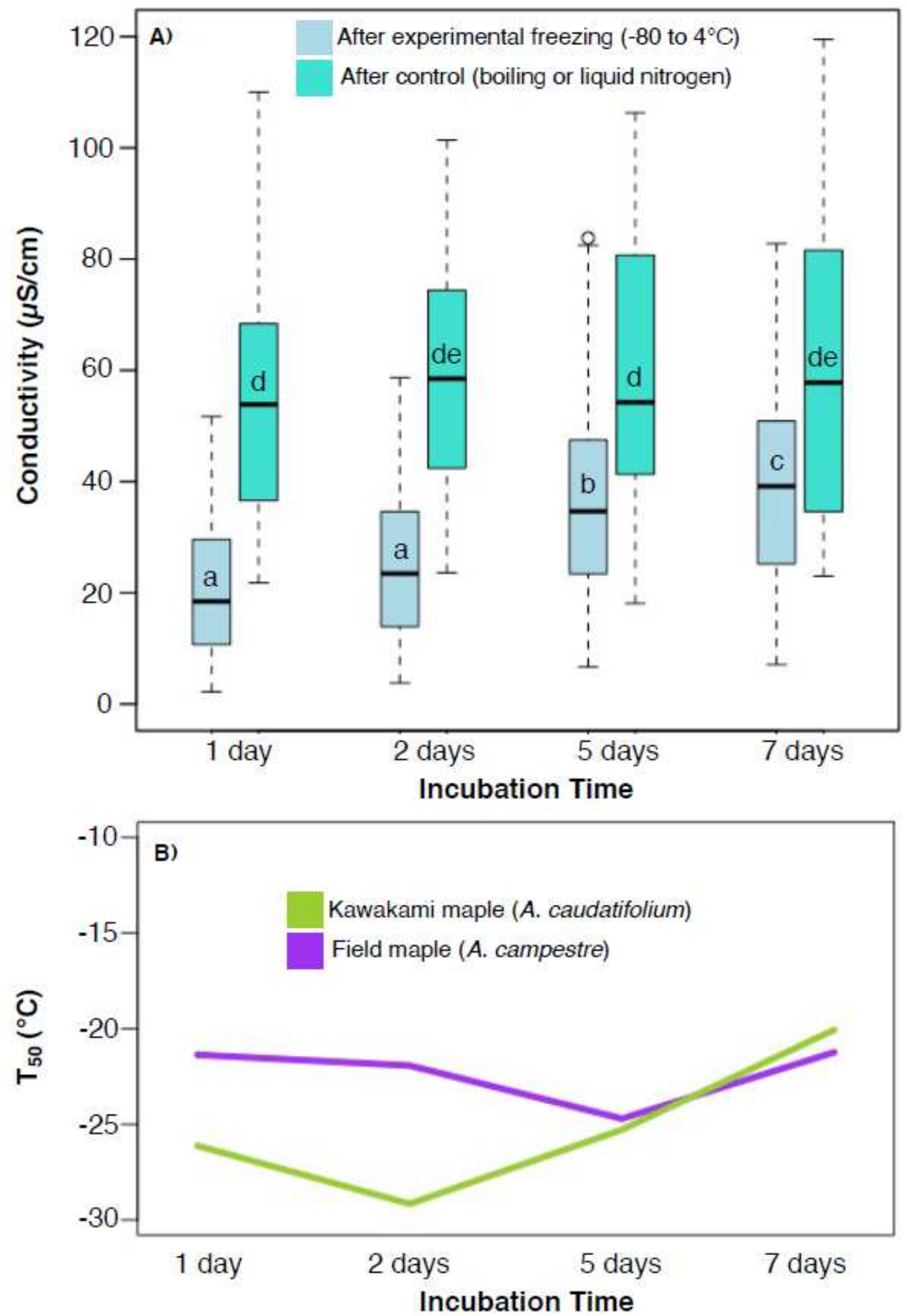

\section{Figure 11}

A) Electrolyte leakage increased gradually over seven days following experimental freezing (light blue), but not exposure to a boiling or liquid nitrogen control (turquoise), with no evidence of a significant difference when conductivity was measured over the first 48 hours after freezing. Lowercase letters indicate significant differences in conductivity measured at different time points at the 0.05 level based on models reported in Additional file 3 panels A (light blue, a-c) and B (turquoise, d-e). B) As a result of 
this pattern, estimates of critical values for cold hardiness (T50) are consistent and reflect species differences when samples were incubated for one or two days, but not when they were incubated for 5 or 7 days (Additional file 4).

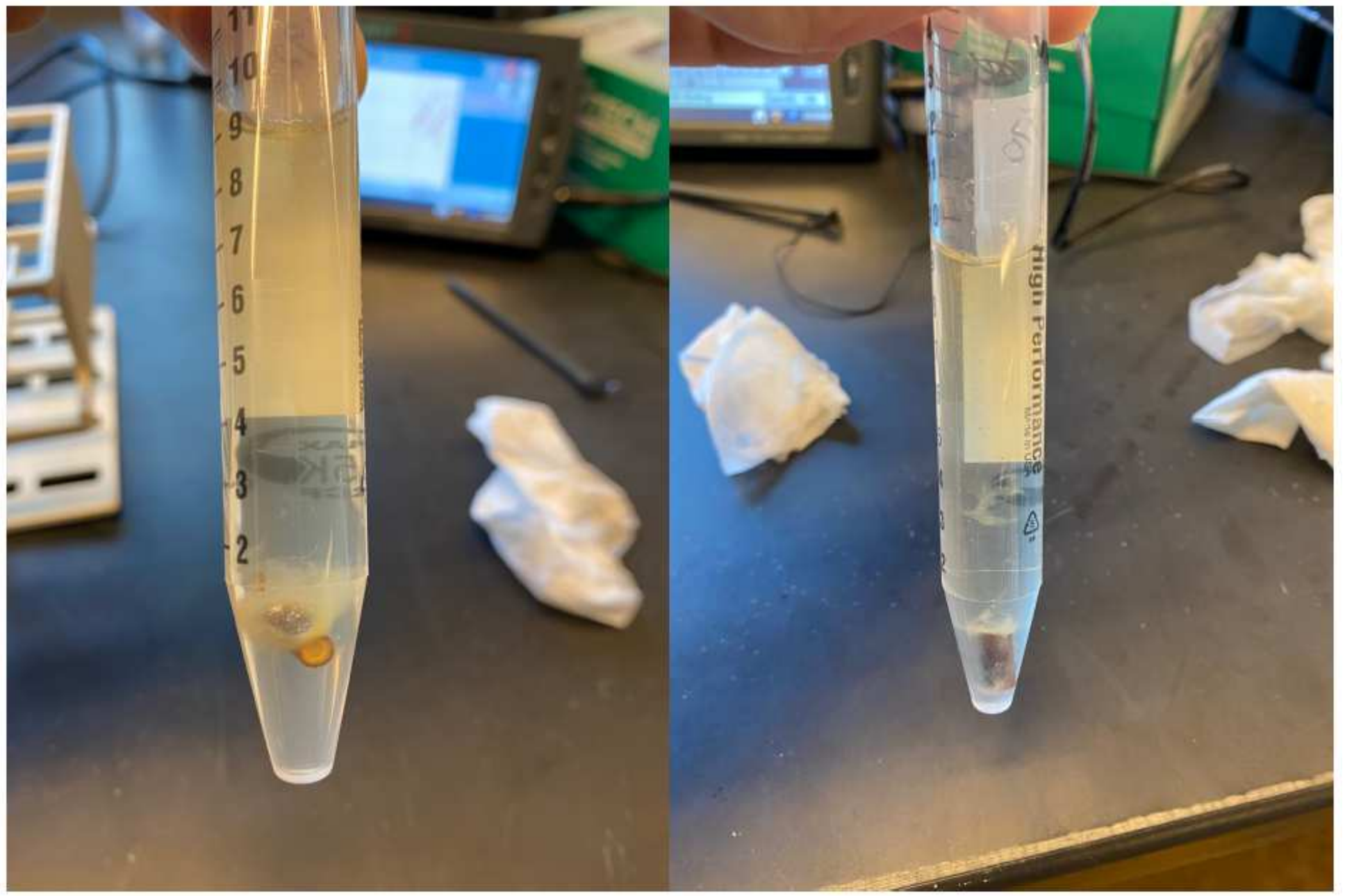

Figure 12

Stem segments incubated for longer than five days following control treatment (boiling or liquid nitrogen immersion) tended to deteriorate, showing evidence of microbial growth.

\section{Supplementary Files}

This is a list of supplementary files associated with this preprint. Click to download.

- renamed495b1.xlsx 